\title{
PRIMEROS RESULTADOS DEL TRABAJO DE INVESTIGACION SOBRE LA TIERRA COMO MATERIAL DE CONSTRUCCION, EN EL IETCC
}

\author{
(FIRST RESULTS FROM RESEARCHES ON EARTH ASA CONSTRUCTION MATERIAL, CARRIED \\ OUT IN THE IETCC)
}

Juan Díaz Romeral, M.a Jesús Guinea, Erhard Rohmer, Julián Salas (V.M.B.C.)

Instituto Eduardo Torroja (CSIC)

\section{RESUMEN}

El trabajo recoge los primeros resultados de la investigación basada en la nueva realidad de las construcciones a base de tierra, teniendo presente su interés ante las nuevas

posibilidades que ofrece a la luz de la crisis energética y, fundamentalmente, por la necesidad de utilización de recursos locales y soluciones viables en paises en vías de desarrollo.

Considerado entre los principales problemas de este tipo de construcciones su limitada capacidad de respuesta ante los agentes atmosféricos -crítica en ambientes húmedos-se pretende establecer parámetros válidos tanto para el comportamiento mecánico, como para su aptitud frente al agua.

Superada una primera fase de clasificación, e identificación de los suelos, se han realizado series de probetas con distintas dosificaciones de cal, cemento, cenizas volantes y otros aditivos químicos, estableciendo parámetros de relación entre distintos coeficientes de forma y comportamiento resistente. De estas dosificaciones se ha estudiado su comportamiento frente a ensayo de goteo y exposición al exterior, en las condiciones atmosféricas de invierno, en Madrid.

\section{SUMMARY}

This work presents the first results from the researches based in the up to date earth constructions, taking into account its interest according to the new possibilities they offer, considering the energetic crisis and, fundamentally, the need to use local resources and feasible solutions in developing countries.

Thinking about the main problems of this kind of construction its short capacity of response to climate conditions-critical in humid environment- we pretend to establish valid parameters for mechanical behaviour so as for resistance under rain.

After a first classification phase and soil identification, series of samples with different dosage, cement, fly ash and other chemical aggregates, were made, establishing relational parameters between different form and resistant behaviour rates. From those dosages, behaviour under dripping and in winter atmosphere in Madrid, were studied.

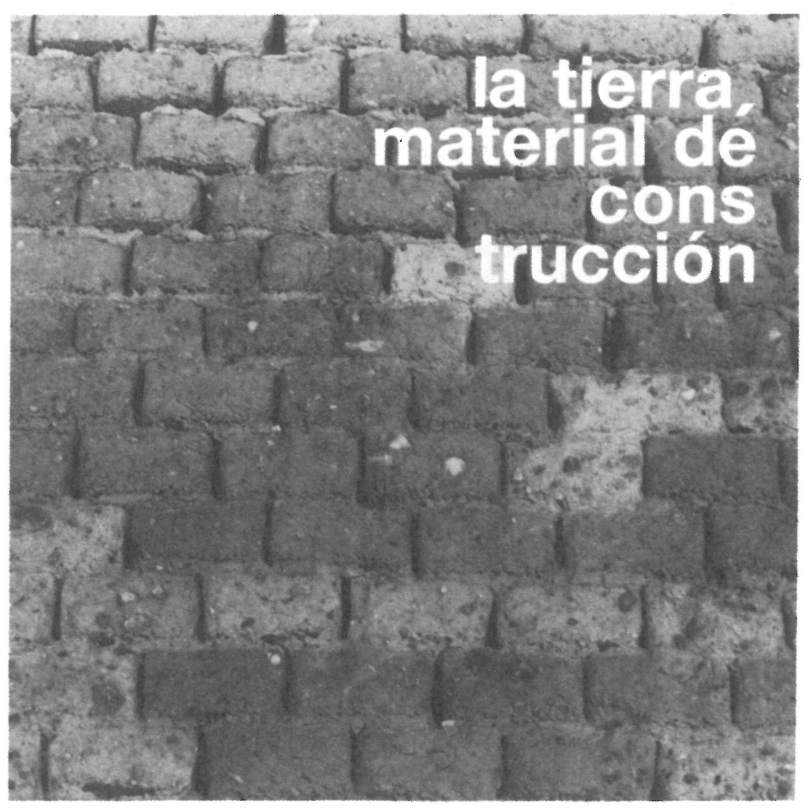

\section{INTRODUCCION}

Uno de los mayores problemas de la aplicación de la tierra en la edificación es su limitada capacidad ante los agentes atmosféricos. Es éste, el argumento tradicional que acompañaba en el largo trayecto que llevaba casi mundialmente hacia el rechazo de este material de construcción.

La patología de la construcción, tanto en los edificios históricos como en las viviendas modernas, evidencia que el diseño arquitectónico / constructivo es factor decisivo para evitar daños por humedades en la edificación. Se inició la investigación, no sólo con la intención de aportar soluciones a medio plazo hacia una aplicación más amplia de este material, incluso para espacios hasta ahora negados para las construcciones de tierra como son las células sanitarias, o en general, los espacios húmedos de las viviendas. 
El inicio del trabajo se enfrenta al conocido problema de la falta de parámetros universalmente reconocidos que permitan la intercambiabilidad de experiencias y el seguimiento del proceso por los profesionales. Esta falta de parámetros fiables y escasez de normas, o sólo la falta de comparabilidad de equipos $u$ organización de ensayos, obtiene otra dimensión y se hace más notable cuando se realiza el trabajo desde un centro dedicado básicamente a la investigación del cemento y a la patologia de las construcciones de hormigón.

Por ello, a parte de pretender reflejar los resultados obtenidos en las series de ensayos, se estima necesario subrayar el propósito de realizar paralelamente:

- La comprobación de las normas ASTM; UNE, etc., en cuanto a su fiabilidad en la investigación -realización de ensayos- de la tierra.

- El desarrollo o aplicación de una metodología fundamentalmente cuantificadora, pero también indicativa, mediante niveles cualitativos específicos.

- El desarrollo de una metodología a través de gráficos de la transformación de datos organoelépticos en numéricos.

- $Y$, en general, hasta que no se disponga de normas o estándares de universal aceptación, el transvase crítico experimental de metodologias propias de la evaluación de la calidad en técnicas de construcción vigentes.

Sin embargo, y junto a este enfoque marcadamente tecnológico, se inicia la investigación, sin olvidar la extraordinaria riqueza del patrimonio de las construcciones de tierra que se manifiesta aun en la Península lbérica, como legado de varias civilizaciones. La selección de los diferentes suelos se efectuó partiendo de las barreras tradicionales utilizadas por los autoconstructores de dos pueblos, Ciadueña y Navapalos, en la provincia de Soria (Castilla La Vieja) cuyos orígenes y composiciones son considerablemente diferentes. Además, se han utilizado dos suelos que se siguen usando masivamente en la fabricación de ladrillos cocidos, los de Alcalá de Henares, en la provincia de Madrid, y de Chiloeches en Guadalajara, así como una mezcla de ambos que nominaremos Alcalá-Chiloeches.

\section{DESCRIPCION DE LOS SUELOS UTILIZADOS}

\subsection{Análisis mineralógico por difracción de rayos $X$}

Realizado sobre los suelos elegidos, se ha llevado a cabo en un difractómetro, modelo PW 4070 para el de Alcalá, y PW 1700 para los restantes,
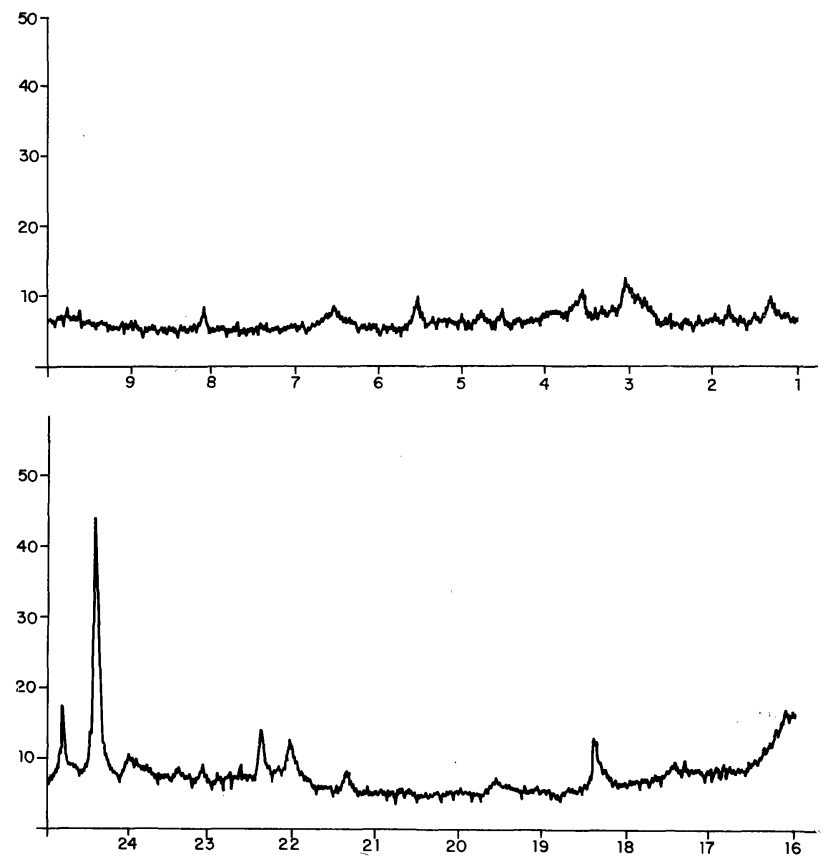

SUELO DE ALCALA DE HENARES

Fig. 1
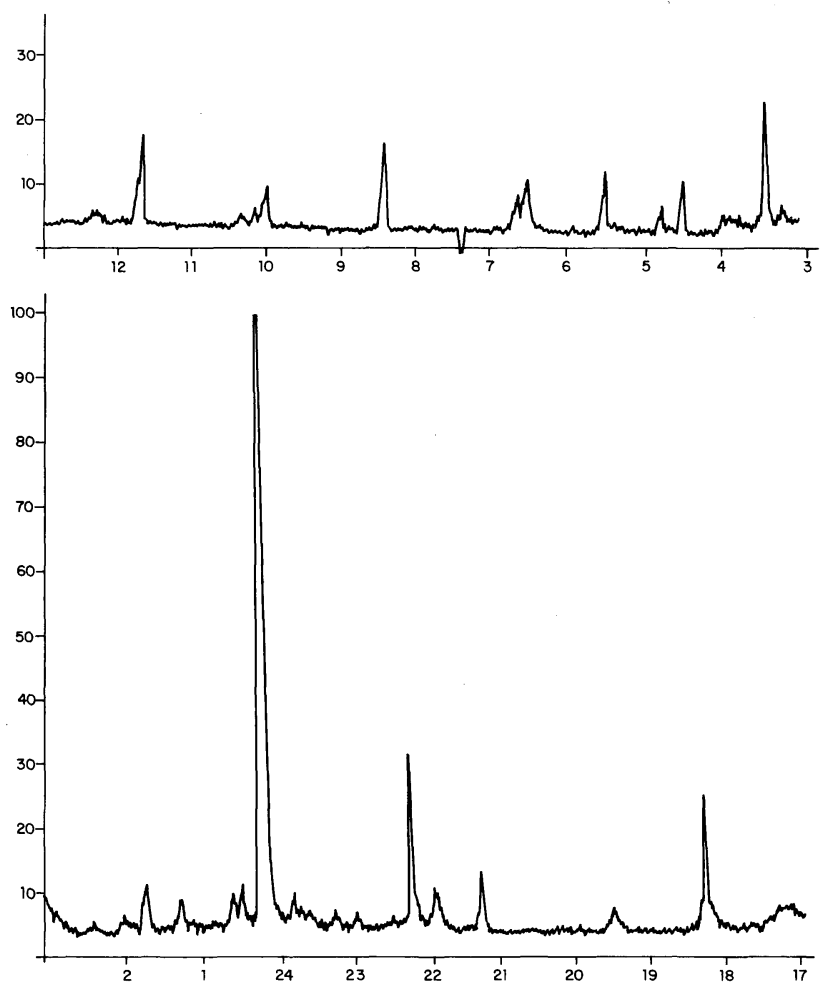

SUELO DE CHILOECHES

Fig. 2 
SUELO de : ALCALA DE HEN:RES. - CHILOECEES


SUELO de : CI:DUU:A.A.
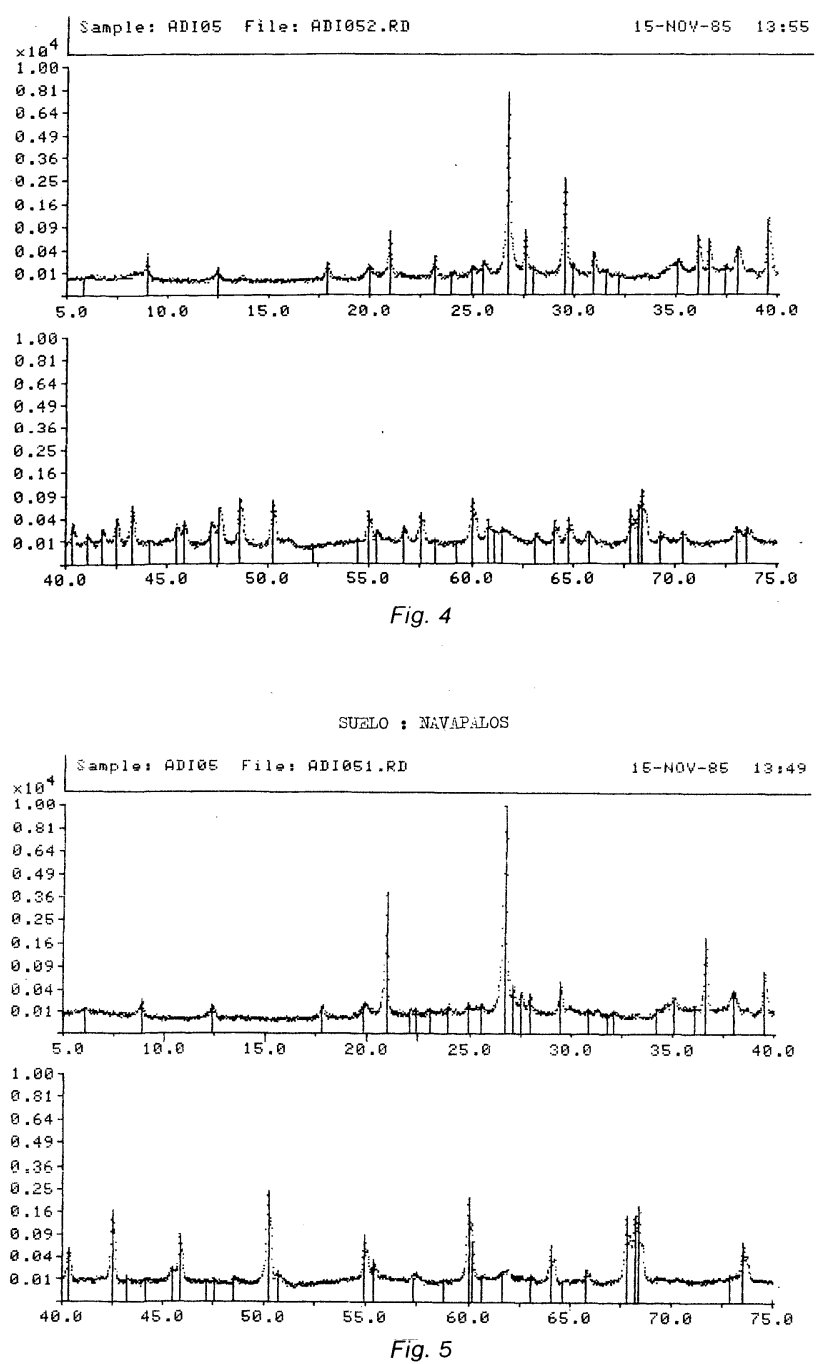

por el método del polvo, utilizando la radiación $\mathrm{K} \alpha$ del cobre y en condiciones standard.

La preparación de la muestra se ha realizado, moliendo el suelo con mortero de ágata hasta la formación de un polvo fino y pasando éste a una prensa semiautomática (marca HERZOG) para la formación de pastillas. La compactación del polvo se realiza sobre anillo de aluminio como portamuestras.

Los suelos de Alcalá de Henares y Chiloeches tienen una composición mineralógica similar a base de mucovita, ilita, cuarzo y en más pequeña proporción feldespatos e indicios de bentonita, si bien las proporciones de los distintos minerales varian notablemente entre una y otra. Asi se observa una mayor presencia de cuarzo en el suelo de Chiloeches. Ver figs. 1 y 2 . El suelo que hemos denominado de Alcalá-Chiloeches fig. 3, contiene cuarzo, calcita, ortoxa, minerales, micáceos y kanditicos, montmorillonita, clorita, vermiculita, hematita, feldespatos en general y palygorskita.

Los suelos de Ciadueña y Navapalos, figuras 4 y 5 respectivamente, tienen una composición mineral semejante entre ellos, contienen cuarzo, calcita, ortosa, minerales micáceos, minerales kanditicos (caolinita), montmorillonita o clorita o vermiculita. Seria necesario obtener los difractogramas de agregado orientado con agua, agregado orientado con glicerina y agregado orientado calcinado a $5000 \mathrm{C}$ dos horas para saber de cuál de los tres últimos compuestos se trata.

\subsection{Composición química}

Se han realizado análisis para determinar la composición química, en los suelos estudiados. La sílice se realizó por gravimetría previa doble insolubilización clorhidrica y posterior purificación fluorhídrica. Los óxidos de aluminio y hierro por espectrometria de absorción atómica. El óxido de titanio por fotocolorimetría con agua oxigenada. Los óxidos de calcio y magnesio por complexometría y los óxidos alcalinos por fotometría de llama. Los resultados de los análisis químicos se recogen en la Tabla n.o 1 de la página siguiente.

\subsection{Estudio granulométrico}

Para la clasificación de los suelos se ha seguido la del «Highway Research Board» (H.R.B.), clasificación basada a la vez en la granulometria y la plasticidad.

Se utiliza un análisis granulométrico simplificado (tamices de $2 \mathrm{~mm}, 0,40 \mathrm{~mm}$ y $80 \mu$ ) así como el. límite líquido y el índice de plasticidad. 
Tabla № 1

ANALISIS QUIMICO

\begin{tabular}{|c|c|c|c|c|c|}
\hline & NAVAPALOS & CIADUE ÑA & $\begin{array}{c}\text { ALCALA } \\
\text { CHILOECHES } \\
\end{array}$ & Alcala & Chiloeches \\
\hline Pérdida por calcinación a $1.100^{\circ} \mathrm{C} \ldots$ & 18,59 & 3,52 & 4,47 & 7,58 & 4,50 \\
\hline Sílice $\left(\operatorname{sio}_{2}\right) \ldots \ldots \ldots \ldots \ldots \ldots \ldots$ & 47,86 & 81,30 & 68,07 & 49,98 & 66,73 \\
\hline Oxido de Hierro $\left(\mathrm{Fe}_{2} \mathrm{O}_{3}\right) \ldots \ldots \ldots \ldots$ & 2,08 & 2,20 & 4,33 & 8,37 & 5,51 \\
\hline oxido de Titanio $\left(\mathrm{TiO}_{2}\right) \ldots \ldots \ldots \ldots$ & 0,29 & 0,39 & 0,60 & 0,76 & 0,86 \\
\hline oxido de Aluminio $\left(\mathrm{Al}_{2} \mathrm{O}_{3}\right) \ldots \ldots \ldots$ & 8,50 & 9,38 & 15,30 & 20,59 & 15,62 \\
\hline Oxido de Magnesio(Mgo)........... & 1,23 & 0,42 & 1,81 & 7,59 & 1,49 \\
\hline oxido de Calcio (Cao).............. & 19,20 & 1,32 & 2,05 & 0,73 & 1,60 \\
\hline Oxido de Sodio $\left(\mathrm{Na}_{2} \mathrm{O}\right) \ldots \ldots \ldots \ldots \ldots$ & 0,09 & 0,22 & 0,70 & 0,69 & 0,40 \\
\hline oxido de Potasio $\left(\mathrm{K}_{2} 0\right) \ldots \ldots \ldots \ldots$ & 1,59 & 1,51 & 2,88 & 3,38 & 2,83 \\
\hline
\end{tabular}

El tamiz de $80 \mu$ permite distinguir los suelos finos (categoria $A-4$ y A-7) de los suelos granulares (cat. A-1, A-2, A-3) según que el porcentaje que pase sea superior o inferior al $35 \%$. Ver tabla de clasificación (H.R.B.):

Clasificación de suelos (H.R.B.)

\begin{tabular}{|c|c|c|c|c|c|c|c|c|c|c|c|}
\hline Clasificación general & \multicolumn{6}{|c|}{$\begin{array}{l}\text { Hasta el } 35 \% \text { de los granos } \\
\text { inferiores a } 80 \mu\end{array}$} & \multicolumn{5}{|c|}{$\begin{array}{l}\text { Más del } 35 \% \text { de los granos } \\
\text { inferiores a } 80 \mu\end{array}$} \\
\hline \multirow{3}{*}{$\begin{array}{l}\text { Porcentaje que pasa en } \\
\text { tamiz de } 2 \mathrm{~mm} . \ldots \ldots \\
\text { tamiz de } 0.40 \mathrm{~mm} . . . \\
\text { tamiz de } 0,80 \mu \ldots \ldots . . .\end{array}$} & At & \multirow[t]{2}{*}{ As } & \multicolumn{4}{|c|}{$A_{2}$} & \multirow[t]{2}{*}{ A. } & \multirow[t]{2}{*}{ As } & \multirow[t]{2}{*}{$A_{0}$} & \multicolumn{2}{|c|}{ Ar } \\
\hline & \begin{tabular}{l|l|}
$A L$ & $A \mathrm{AL}$
\end{tabular} & & $A z-1$ & $\mid A_{2-s}$ & $A 2-6$ & $A_{2-7}$ & & & & $A t-s$ & $A_{t \rightarrow-4}$ \\
\hline & $\left|\begin{array}{r|r|}<50 \\
<30 \\
<15\end{array}\right|<250$ & $\begin{array}{l}251 \\
<10\end{array} \mid$ & $<35$ & $<35$ & $\leqslant 35$ & $\mid<35$ & $>36$ & $>36$ & $>36$ & $>36$ & $>36$ \\
\hline $\begin{array}{l}\text { Caracteristicas de la frac- } \\
\text { ción que pasa por un } \\
\text { tamiz de } 2 \mathrm{~mm} \text {................... }\end{array}$ & & & & & & & & & & & \\
\hline - Indice de plasticidad. & & $\begin{array}{l}\text { sible } \\
\text { medir }\end{array}$ & $<10$ & $\mid<10$ & $>11$ & $>11$ & $<10$ & $<10$ & $>11$ & $\begin{array}{l}\geqslant 11 \\
1 P< \\
w L-30\end{array}$ & $\left|\begin{array}{l}>11 \\
1 r> \\
w L-30\end{array}\right|$ \\
\hline - Limite liquido ....... & $\mid \begin{array}{l}\text { imposible } \\
\text { determinar }\end{array}$ & - & $\leqslant 40$ & $>41$ & $\leqslant 40$ & $01>41$ & $<40$ & $>41$ & $<40$ & $>41$ & $>41$ \\
\hline - Indice de grupo ....... & 0 & 0 & & 0 & & $\leqslant 4$ & $\leq 8$ & $\leqslant 12$ & $<16$ & $<20$ & $<20$ \\
\hline - Denominación general & $\begin{array}{l}\text { Cantos } \\
\text { Gravas } \\
\text { Arenas }\end{array}$ & $\begin{array}{c}\text { Are- } \\
\text { na } \\
\text { fina }\end{array}$ & $\begin{array}{l}\text { Mezc } \\
\text { mosa } \\
\text { aren }\end{array}$ & $\begin{array}{l}\text { cla de } \\
\text { as o or } \\
\text { aas lim } \\
\text { cill }\end{array}$ & $\begin{array}{l}\text { e grava } \\
\text { cillosas } \\
\text { nosas } \\
\text { losas }\end{array}$ & $\begin{array}{l}\text { vas li- } \\
\text { vas con } \\
\text { o ar- }\end{array}$ & Sue & $\begin{array}{l}\text { elos } \\
\text { osos }\end{array}$ & Suel & los arci & illosos \\
\hline
\end{tabular}

Los suelos empleados dieron los resultados siguientes:

- Alcalá, pasa por el tamiz de $80 \mu$. . . 97,3 \%

- Chiloeches, pasa por el tamiz de $80 \mu \quad 74,2 \%$

- Alcalá-Chiloeches (al 50\%), pasa por

el tamiz de $80 \mu \ldots \ldots \ldots \ldots \ldots \ldots \ldots 50,4 \%$

- Ciadueña, pasa por el tamiz $80 \mu$. . . 55,1\%

- Navapalos, pasa por el tamiz $80 \mu \ldots \quad 61,5 \%$

Puede afirmarse que todos son suelos finos, ya que el porcentaje de los mismos que pasa por el tamiz de $80 \mu$ es superior al $35 \%$.
Teniendo en cuenta los valores obtenidos en la tabla 3 (pág. 11), se han estudiado distintas relaciones.

Primero, tomando como variable independiente el contenido de arcilla de cada uno de los suelos, es decir, las partículas más finas de los mismos, con diámetros inferiores a $2 \mu$ y que son los elementos más activos de los constituyentes de los suelos, ya que las partículas superiores a $2 \mu$ no actúan más que de modo análogo al de los áridos en el hormigón, y como variable dependiente: 1. - el Indice de Plasticidad; 2. - la Humedad Natural; 3. - el Límite Líquido; 4. - la Resistencia a Compresión. Vemos en la fig. 6 que ajustando por minimos cuadrados las rectas que obtendriamos con la serie de puntos, se obtienen unos coeficientes de correlación aceptables, por lo que podemos decir que existe una correlación entre dichos valores.

Del mismo modo hacemos la relación, tomando como variable independiente el contenido de finos de cada uno de los suelos: 1 . - el Indice de Plasticidad; 2. - el Límite Plástico; 3. - el Límite Liquido; 4. - la Retracción. Comprobando (ver fig. 7) que existe también una relación.

\section{FUNCIONES OBTENIDAS:}

* INDICE DE PLASTICIDAD - ARCILLA

$y=-1,50+0,64 x$

$C=0,99$ $E=0,42$

* humedad nAtURAL - ARCILLA

$y=0,44+0,10 x$

$C=0,96$

$\mathrm{E}=0,25$ 

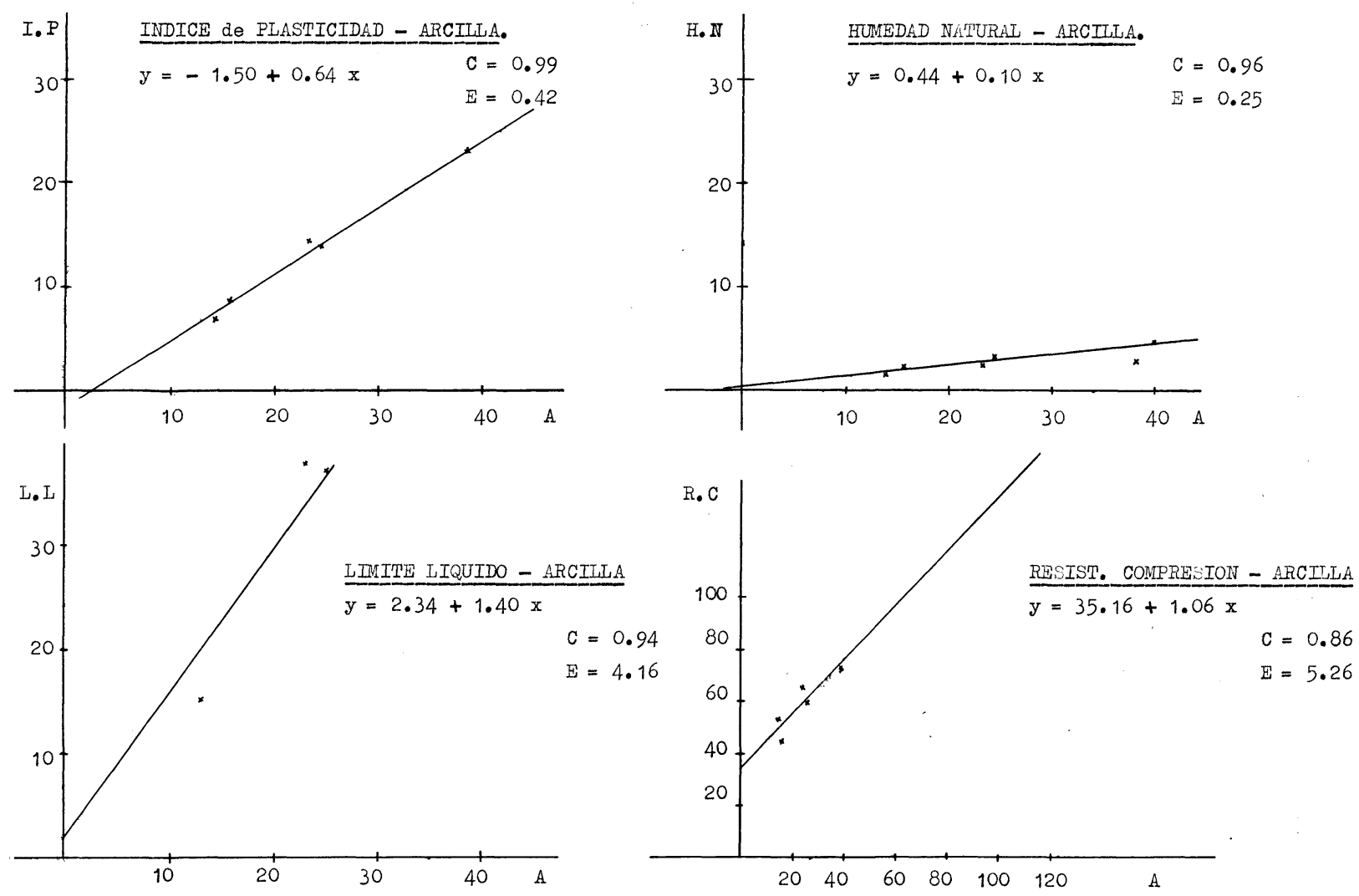

Fig. 6
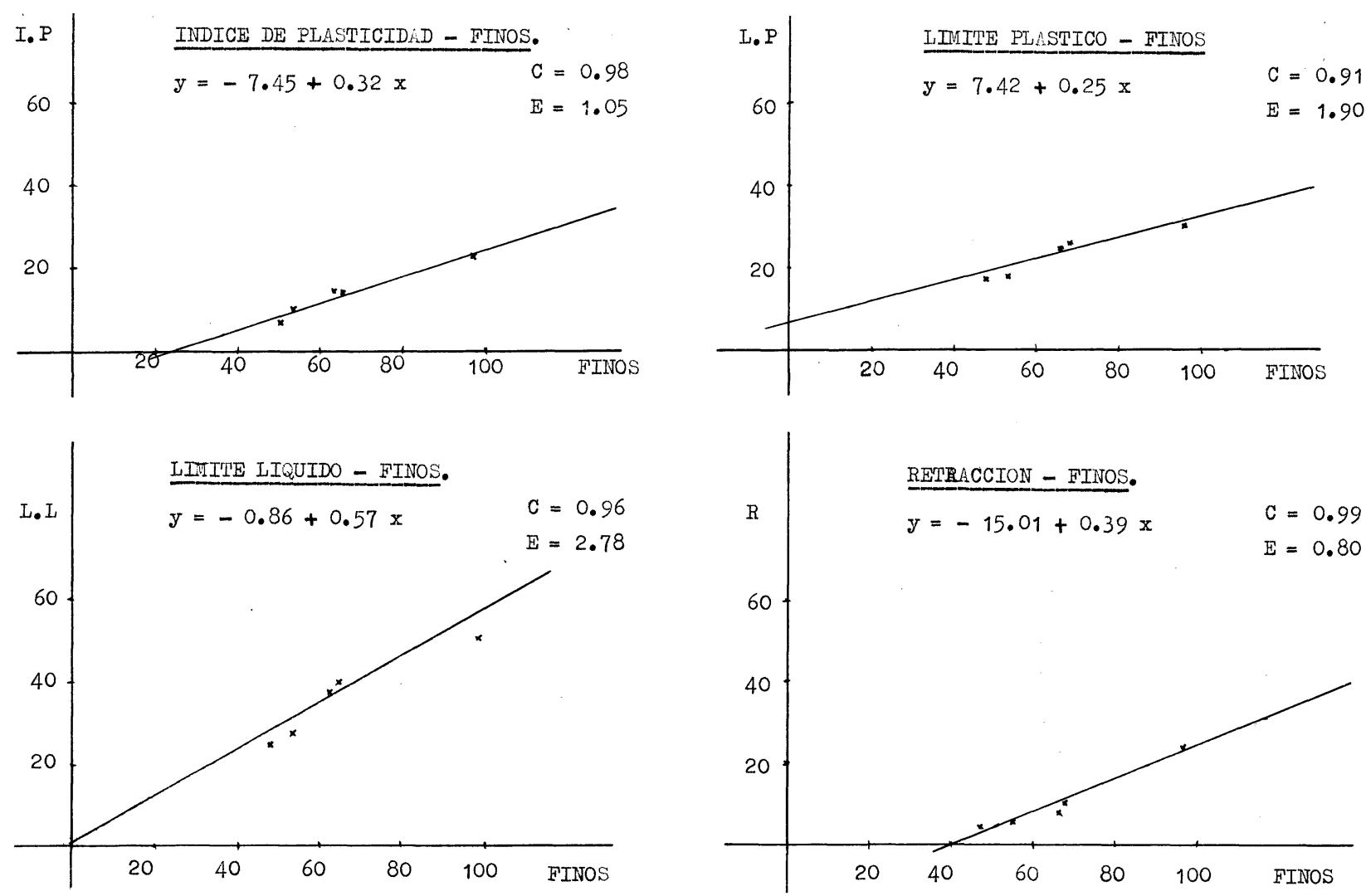

Fig. 7 
* LIMITE LIQUIDO - ARCILLA

$y=2,34+1,40 x$

* RESISTENCIA A COMPRESION - ARCILLA

$y=35,16+1,06 x$

$C=0,86$

$\mathrm{E}=5,28$

* INDICE DE PLATICIDAD-FINOS

$y=-7,45+0,32 x$

$C=0,98$

$E=1,05$

* LIMITE PLASTICO - FINOS

$y=7,42+0,25 x$

$\mathrm{C}=0,91$

$\mathrm{E}=1,90$

* LIMITE LIQUIDO - FINOS

$y=-0,86+0,57 x$

* RETRACCION-FINOS

$y=-15,01+0,39 x$

$C=0,96$

$\mathrm{E}=2,78$
La siguiente división según el índice de plasticidad, permite distinguir entre suelos limosos $\left(I_{p} \leqslant\right.$ 10 , grupos $A-4$ y $A-5)$ y arcillosos $\left(I_{p} \geqslant 11\right.$, grupo $A-6$ y $A-7)$.

El valor del límite líquido, separará el grupo A-4 del A-5 y el A-6 del A-7.

- Suelo de ALCALA: Suelo franco-limo-arcilloso

Limite plástico........ 30,25\%

Limite liquido .......... 53,00\% $>41 \rightarrow \mathrm{A}_{7}$

Indice de plasticidad ... 22,70\% $>11 \rightarrow \mathrm{A}_{6} \mathrm{O}^{\mathrm{A}} \mathrm{A}_{7}$

- Suelo de CHILOECHES: Suelo franco-arcilloarenoso

Límite plástico....... 26,80 \%

Limite liquido ......... 40,80 \% $<41 \rightarrow A_{6}$

Indice de plasticidad ... 14,00\% $>11 \rightarrow \mathrm{A}_{6}$ ó $\mathrm{A}_{7}$

- Suelo de ALCALA-CHILOECHES: Suelo francoarenoso

Límite plástico....... 18,6\%

Limite líquido ........ 27,2\% $<40 \rightarrow A_{4}$

Indice de plasticidad $\ldots \quad 8,6 \%<10 \rightarrow A_{5}$ ó $A_{4}$

- Suelo de CIADUEÑA: Suelo franco-arenoso

Limite plástico........ 18,3\%

Limite liquido ......... 25,2\% $\quad<40 \rightarrow \mathrm{A}_{4}$

Indice de plasticidad . . 6,9\% $<10 \rightarrow A_{4}$ ó $A_{5}$

- Suelos de NAVAPALOS: Suelo franco-arcilloarenoso

Limite plástico........ 24,1\%

$C=0,99 \quad$ Limite líquido $\ldots \ldots \ldots .38,5 \%<40 \rightarrow A_{6}$

$E=0,80 \quad$ Indice de plasticidad ... 14,4\% $>11 \rightarrow A_{6}$ ó $A_{7}$

Tabla 2

\begin{tabular}{|c|c|c|c|c|c|c|c|c|c|}
\hline & $\mu, \mathrm{m}$ & $10^{3}$ & 5. $10^{2}$ & 2. $10^{2}$ & 5. 10 & 20 & 2 & $<2$ & \\
\hline & $\mathrm{mm}$. & 1 & 0.5 & 0.2 & 0.05 & 0.02 & 0.002 & 0.002 & \\
\hline \multicolumn{2}{|c|}{ Retenidos parciales } & - & 0.1 & 0.4 & 3.0 & 10.8 & 47.4 & 38.3 & \multirow{2}{*}{$\begin{array}{l}\text { suelo: ALCALA de HEHARES } \\
\text { FrR NACO - LIMO - ARCILLOSO }\end{array}$} \\
\hline \multicolumn{2}{|c|}{ Retenidos acumulados } & - & 0.1 & 0.5 & 3.5 & $14 \cdot 3$ & 61.7 & 100.0 & \\
\hline \multicolumn{2}{|l|}{ Ret. parciales. } & 1.7 & 8.1 & 24.4 & 26.6 & 7.5 & $15 \cdot 7$ & 16.0 & \multirow{2}{*}{$\begin{array}{l}\text { suelo: LCALA - CHILOECHES } \\
\text { FRENCO - RENOSO }\end{array}$} \\
\hline Ret. acumulado. & & 1.7 & 9.8 & 34.2 & 60.8 & 68.3 & 84.0 & 100.0 & \\
\hline Ret. parciales. & & 0.1 & 1.2 & 5.6 & 28.3 & 14.0 & 26.7 & 24.1 & \multirow{2}{*}{$\begin{array}{l}\text { suelo: CHILOECHES } \\
\text { FRAIICO - HCIELLO AREIOSO }\end{array}$} \\
\hline \multicolumn{2}{|l|}{ Ret. acumulado. } & 0.1 & 1.3 & 6.9 & 35.2 & 49.2 & 75.9 & 100.0 & \\
\hline \multicolumn{2}{|l|}{ Ret. parciales. } & 0.2 & 0.2 & 12.0 & 47.2 & 16.6 & 10.1 & 13.7 & \multirow{2}{*}{$\begin{array}{l}\text { suelo: CIADURNA } \\
\text { FRAITCO - AFENOSO }\end{array}$} \\
\hline \multicolumn{2}{|l|}{ Ret. acumulado. } & 0.2 & 0.4 & 12.4 & 59.6 & 76.2 & 86.3 & 100.0 & \\
\hline Ret. parciales. & & 0.5 & 3.6 & 16.4 & 23.5 & 11.9 & 20.5 & 23.6 & \multirow{2}{*}{$\begin{array}{l}\text { suelo: HAVAFLIOS } \\
\text { FRINCO - ARCILLO - ARENOSO }\end{array}$} \\
\hline \multirow{2}{*}{\multicolumn{2}{|c|}{ Ret. acumulado. }} & 0.5 & 4.1 & 20.5 & 44.0 & 55.9 & 76.4 & 100.0 & \\
\hline & & $\begin{array}{c}\text { arena } \\
M_{0} G\end{array}$ & $\begin{array}{c}\text { arene } \\
G\end{array}$ & $\begin{array}{c}\text { arene } \\
\text { II }\end{array}$ & $\begin{array}{c}\text { arena } \\
\mathrm{F}\end{array}$ & $\begin{array}{c}\text { arena } \\
\mathrm{K} . \mathrm{F}\end{array}$ & limo & arcilla & \\
\hline
\end{tabular}

M.G. - muy gruesa (2-1 mm). G. - gruesa $(1-0.5 \mathrm{~mm})$. M. - inedia $(0.5-$ c. $\mathrm{mm})$. F. - fina $(0.2-0.05 \mathrm{rmm})$. M.F. - muy fina (0.05-0.02 mm). 
En la Tabla n.o 2 se recogen los valores retenidos parciales y acumulados por la serie de tamices constituida por la clasificación en: arena muy gruesa; gruesa; media; fina; muy fina; limo y arcilla. Los valores correspondientes a las cinco tierras estudiadas se representan en la fig. 8.

Por último, en la Tabla n.o 3 se recogen el conjunto de valores cuantitativos capaces de delimitar cada uno de los suelos estudiados, siguiendo las directrices de la norma DIN 18.952.

\section{ENSAYOS MECANICOS}

Desde el punto de vista de los resultados de los ensayos resistentes, tres han sido los objetivos fundamentales de este trabajo.

I. Buscar relaciones cuantitativas fiables entre resultados a compresión en dos tipos de probetas distintas (influencia de la forma) y entre dos formas diferentes de trabajo (compresión - flexotracción).

Tabla 3

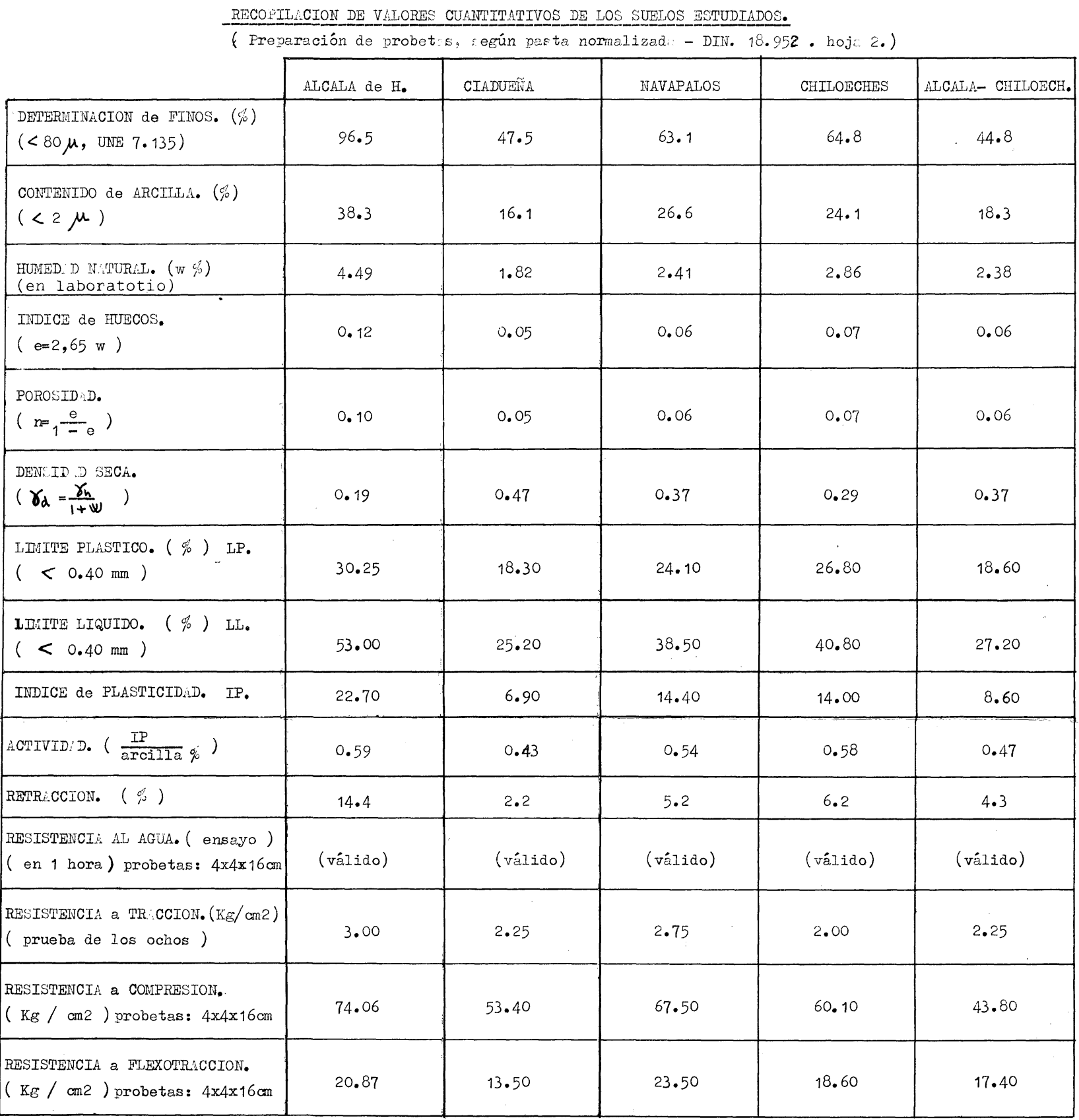


Tabla 4

IDENTIFICACION DE MEZCLAS MEDIANTE DOS NUMEROS: UNO PARA CADA PROCEDENCIA DE SUELO Y OTRO PARA CADA COMPOSICION CONCRETA



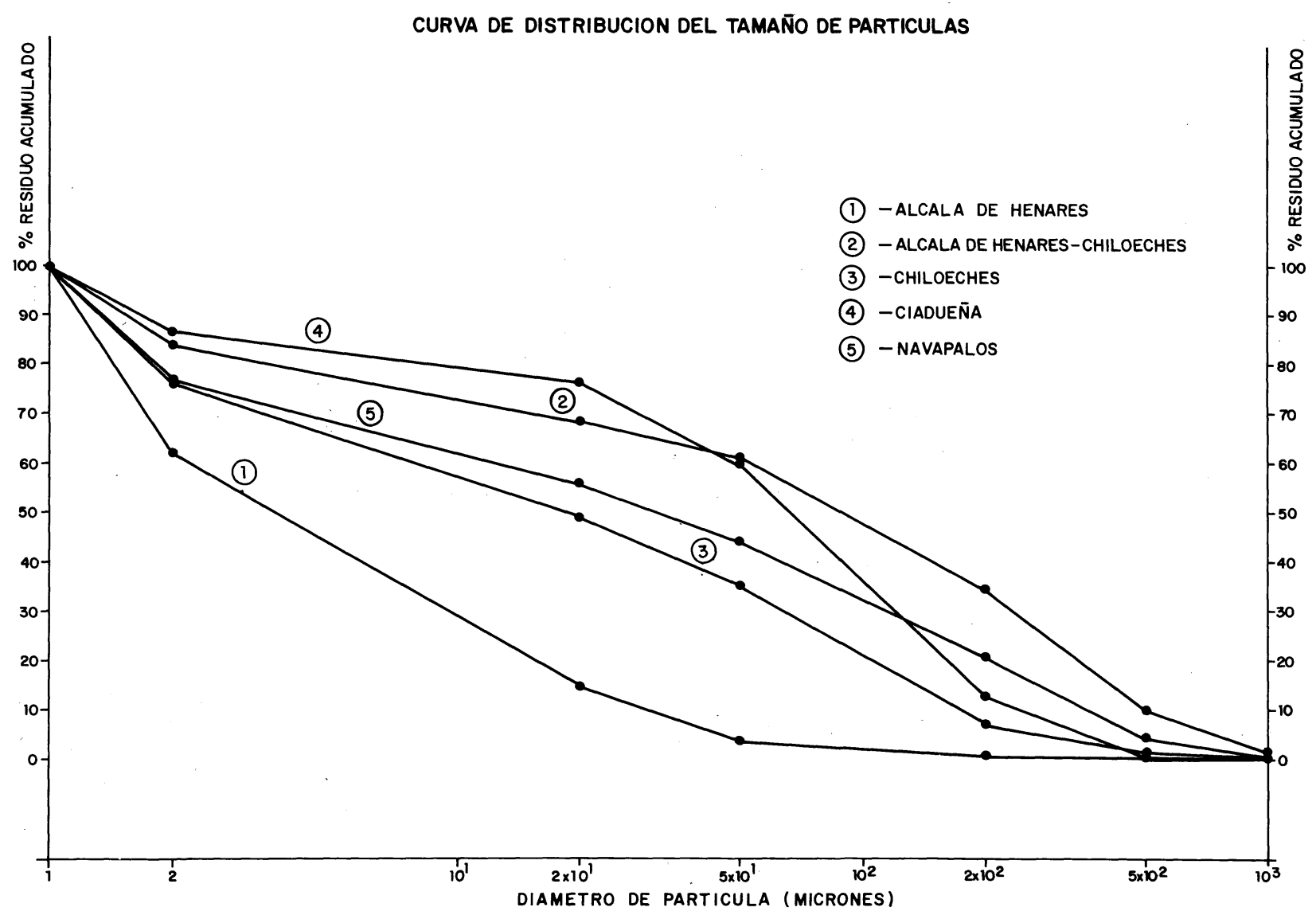


II. Definir unos niveles cualitativos del comportamiento resistente de las distintas mezclas.

III. Tratar de interrelacionar resistencias mecánicas (comportamiento estructural) con composiciones de mezclas capaces de mejorar la resistencia a las agresiones del agua y la intemperie (comportamiento funcional).

La nomenclatura empleada en la designación de los diferentes suelos o de sus combinaciones con cal, cemento, cenizas volantes o una determinada sustancia impermeabilizable, es la que de forma esquemática se recoge en la Tabla 4, mediante dos números.

En el proceso de fabricación de las probetas, se ha seguido principalmente lo establecido por la antigua norma DIN 18952 hoja 2, tanto en lo que se refiere a la plasticidad normalizada como al proceso de secado. El contenido de agua se comprobó constantemente mediante la toma de muestras en pesasustancias y su posterior secado y control de peso.

\subsection{Ensayos a compresión y flexotracción}

El primero de los objetivos enunciados es deducible de las figuras 9 y 10 . En la primera de ellas, en abcisas se han representado los valores medios de series de tres probetas de $7 \times 7 \times 7 \mathrm{~cm}$, rotas a compresión, expresados en $\mathrm{kp} / \mathrm{cm}^{2}$ y en ordenadas iguales resultados para series de seis valores, correspondientes a las porciones de probetas de $4 \times 4 \times 16 \mathrm{~cm}$ tras haber sido sometidas a rotura a flexotracción. Los resultados corresponden en ambos casos a diez mezclas distintas en base al suelo de Alcalá-Chiloeches

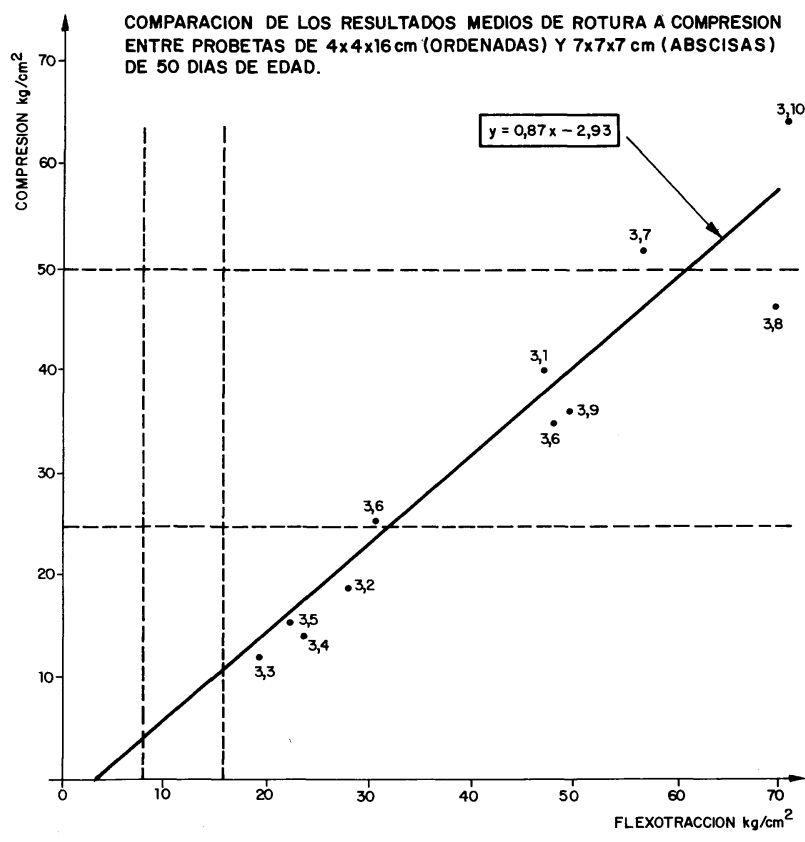

Fig. 9

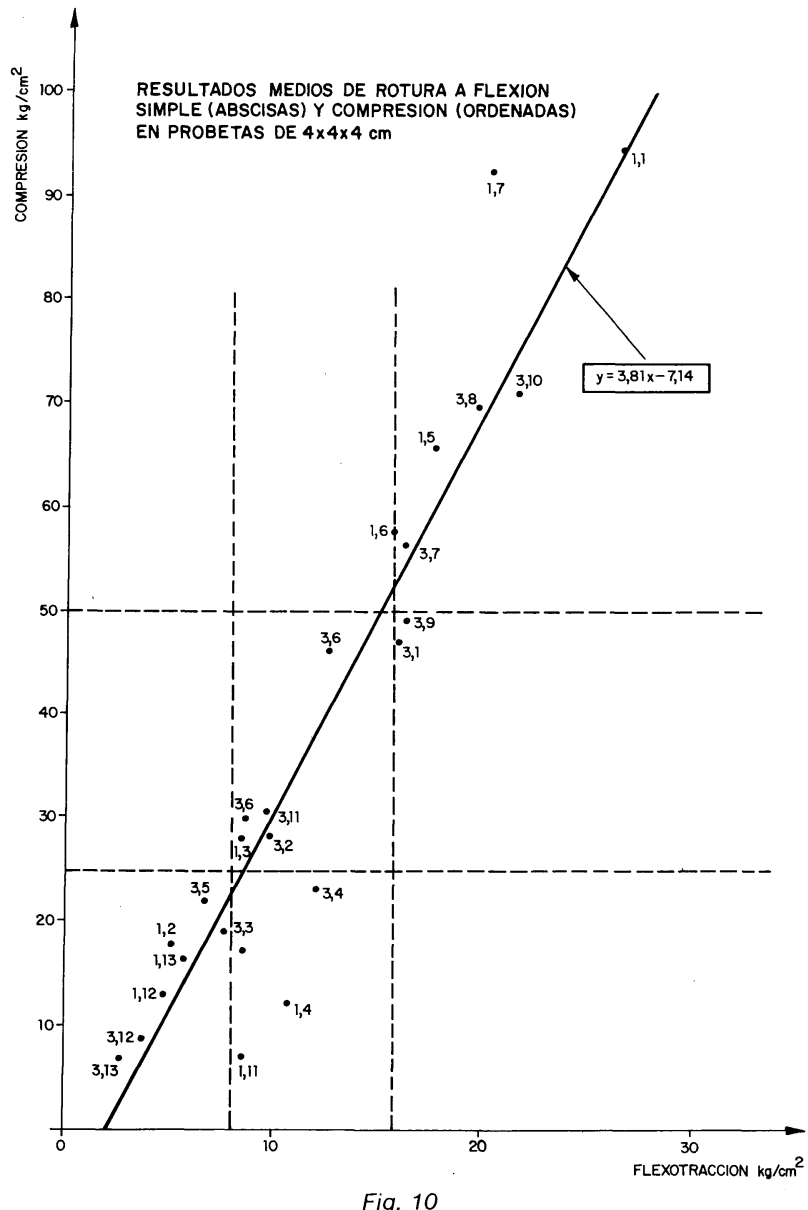

estudiado, medidos sobre probetas realizadas en condiciones semejantes y curadas durante 50 dias en ambiente de laboratorio ( $\simeq 170 \mathrm{C}$ y $50 \div 60 \%$ de humedad) que antes de ensayar se sometieron durante $24 \mathrm{~h}$ a $50 \circ \mathrm{C}$ de temperatura. Mediante ajuste por minimos cuadrados se ha determinado la ecuación:

$$
y=0,87 x-2,93
$$

que permite determinar de forma práctica los valores de la resistencia a compresión en probetas de $7 \times 7 \times 7 \mathrm{~cm}$. conociendo la resistencia a compresión en $4 \times 4 \times 16 \mathrm{~cm}$ y viceversa.

De forma semejante (fig. 10) se ha determinado la recta:

$$
y=3,81 x-7,14
$$

como ajuste de veintiséis puntos, valores medios de ensayos a compresión y flexotracción en probetas de $4 \times 4 \times 16 \mathrm{~cm}$ correspondientes a una amplisima gama de calidades, para suelos de Navapalos y Alcalá-Chiloeches.

\subsection{Niveles cualitativos}

Siguiendo los criterios generales apuntados en la introducción referentes a la clasificación me- 
Tabla 5

RESISTENCIA A COMPRESION A 50 DIAS $\left(\mathrm{kP} / \mathrm{cm}^{2}\right)$

\begin{tabular}{|c|c|c|c|c|c|}
\hline & BA JA & MEDIA & ALTA \\
\hline & & & $\sigma_{c}<25$ & $25<\sigma_{c}<50$ & $50<\sigma_{c}$ \\
\hline \multirow{3}{*}{ 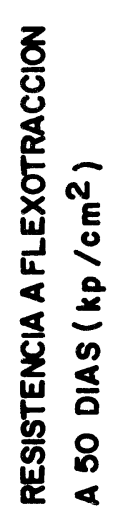 } & $\underset{\leftarrow}{\mathbb{L}}$ & $\begin{array}{c}0 \\
\wedge 1 \\
0 \\
6\end{array}$ & A & B & C \\
\hline & $\begin{array}{l}\frac{a}{0} \\
\frac{u}{2}\end{array}$ & $\begin{array}{l}\underline{\varphi} \\
v_{0} \\
\sigma \\
v \\
v \\
\infty\end{array}$ & A & B & B \\
\hline & 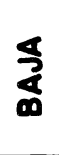 & $\begin{array}{l}0 \\
\hat{b} \\
\hat{\infty}\end{array}$ & A & A & A \\
\hline
\end{tabular}

diante niveles cualitativos, en base a la experiencia sobre necesidades de capacidad resistente «normales» en las aplicaciones de la tierra en "viviendas de muy bajo costo" (una planta de altura y vanos de escasa luz), se proponen tres niveles cualitativos: calidades baja, media y alta teniendo presente los resultados de los ensayos recogidos en la Tabla 5 y representados por las zonas $\mathrm{A}, \mathrm{B}$ y $\mathrm{C}$ respectivamente.

De forma acorde con estos criterios, en las Tablas 6 y 7 se recogen los resultados a compresión y flexotracción de los distintos suelos ensayados y representados en las figuras 5 y 6 .

\subsection{Influencia entre composición y comportamiento mecánico}

El tercer objetivo pretendido, la relación o influencia entre comportamiento mecánico y distintas composiciones cuantitativa / cualitativa, es sin duda más fácil de acotar.

Tabla 6

\begin{tabular}{|c|c|c|c|c|c|c|c|c|c|c|c|c|c|c|}
\hline & \multirow{2}{*}{\begin{tabular}{|c|}
$100 \%$ \\
1 \\
\end{tabular}} & \multicolumn{3}{|c|}{$\overline{C A I}$} & \multicolumn{3}{|c|}{ CENENTO } & \multicolumn{3}{|c|}{ CENIZA } & \multicolumn{3}{|c|}{ REMO } & \\
\hline & & 2 & 3 & 4 & 5 & 6 & 7 & 8 & 9 & 10 & 11 & 12 & 13 & \\
\hline \multirow{2}{*}{ ALTA } & 1.1 & & & & 1.5 & & 1.7 & & & & & & & 1.- NAV. \\
\hline & & & & & & & & 3.8 & & 3.10 & & & & 2. - CIID. \\
\hline \multirow{2}{*}{ MEDIA } & & & 1.3 & & & 1.6 & & 1.8 & 1.9 & 1.10 & & & & 3. - ALC-CH \\
\hline & 3.1 & 3.2 & & & & 3.6 & 3.7 & & 3.9 & & 3.1 & & & 1.- \\
\hline \multirow{2}{*}{ BAJA } & & 1.2 & & 1.4 & & & & & & & 1.11 & 1.12 & 1.13 & $2 .-$ \\
\hline & & & 3.3 & 3.4 & 3.5 & & & & & & & 3.12 & 3.13 & 3.- \\
\hline
\end{tabular}

Tabla 7

RESISTENCIA de PROBETAS $(4 \times 4 \times 16)$ a FLEXOTRALCION.

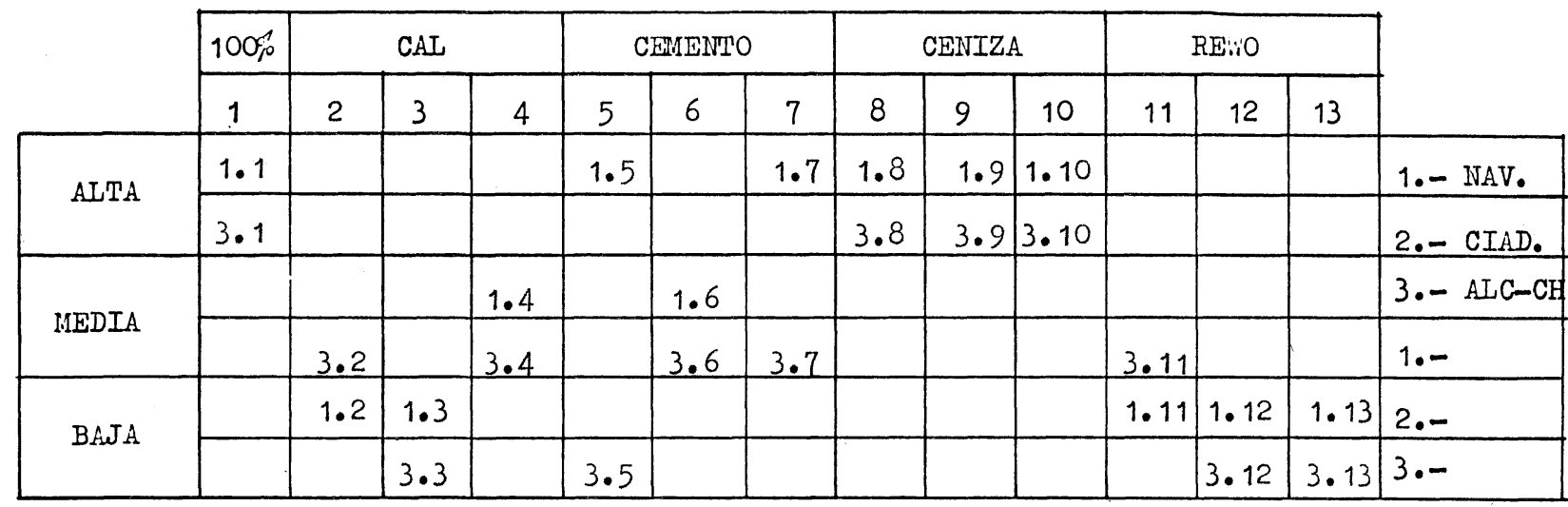


INFLUENCIA EN LAS CARACTERISTICAS RESISTENTES DEL SUELO DE NAVAPALOS CON DISTINTAS ADICIONES (CAL, CENIZAS VOLANTES, CEMENTO PA 350 Y UN PRODUCTO IMPERMEABILIZANTE -REWO-) EN PROPORCIONES EN PESO DEL 5,10 Y $15 \%$

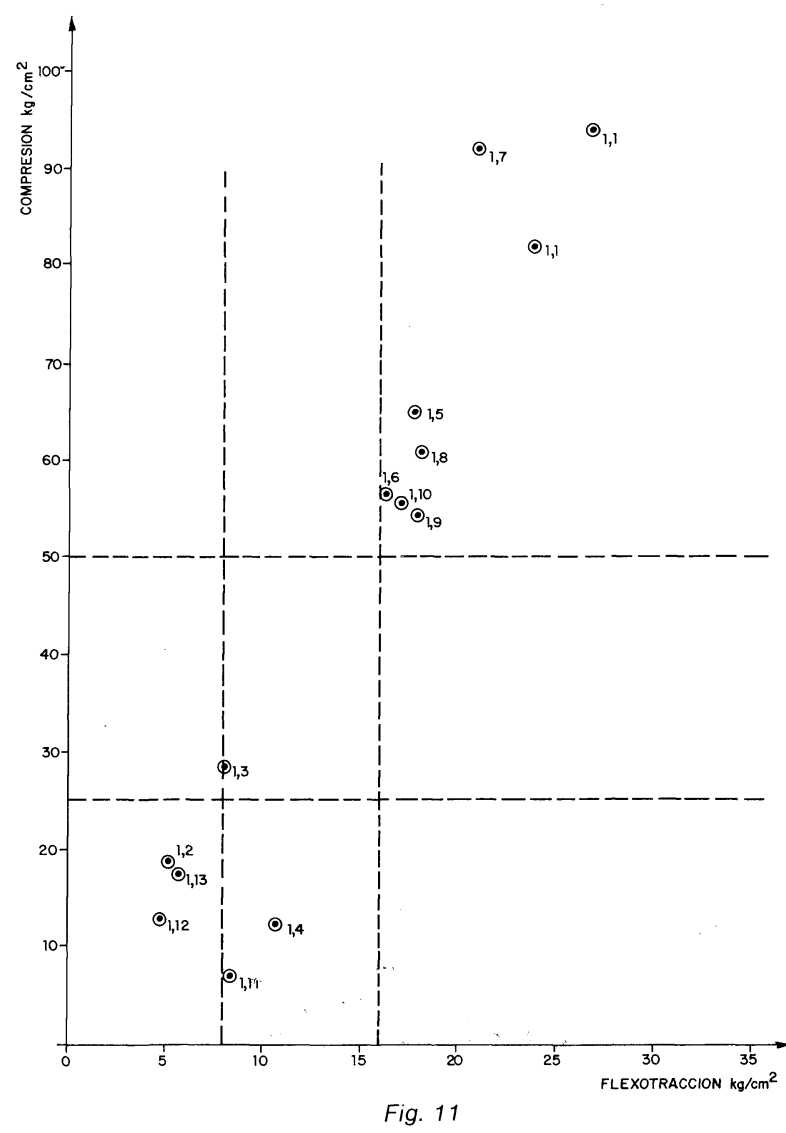

De los suelos de Navapalos y Alcalá - Chiloeches estudiados, se han realizado series de tres probetas de $7 \times 7 \times 7 \mathrm{~cm}$. en cada caso y en las condiciones ya mencionadas, en las que partiendo de cada suelo sin adición alguna, se han añadido en proporciones en peso del 5\%; $10 \%$ y $15 \%$ respectivamente, cada uno de los siguientes materiales:

a) Cemento portland con hasta un $19 \%$ de adiciones, PA-350.

b) Cenizas volantes silico-calcáreas procedentes de la térmica de Soto de Ribera.

c) Cal apagada.

d) El impermeabilizante de mercado, "Adogen» -dimetil diesterol - cloruro amónico- (en proporciones, sin duda inadecuadas, al objeto de provocar en este caso especifico un efecto distorsionador en las propiedades resistentes).

En todos los casos se ha seguido la nomenclatura recogida en la Tabla n.o 4.

Del análisis de las figuras 11 y 12 se deduce que:
INFLUENCIA EN LAS CARACTERISTICAS RESISTENTES DEL SUELO ALCAL-CHILOECHES CON DISTINTAS ADICIONES (CAL, CENIZA VOLANTE, CEMENTO BA-350 Y UN PRODUCTO IMPERMEABILIZANTE -REWO-) EN PROPORCIONES EN PESO DEL 5,10 Y $15 \%$

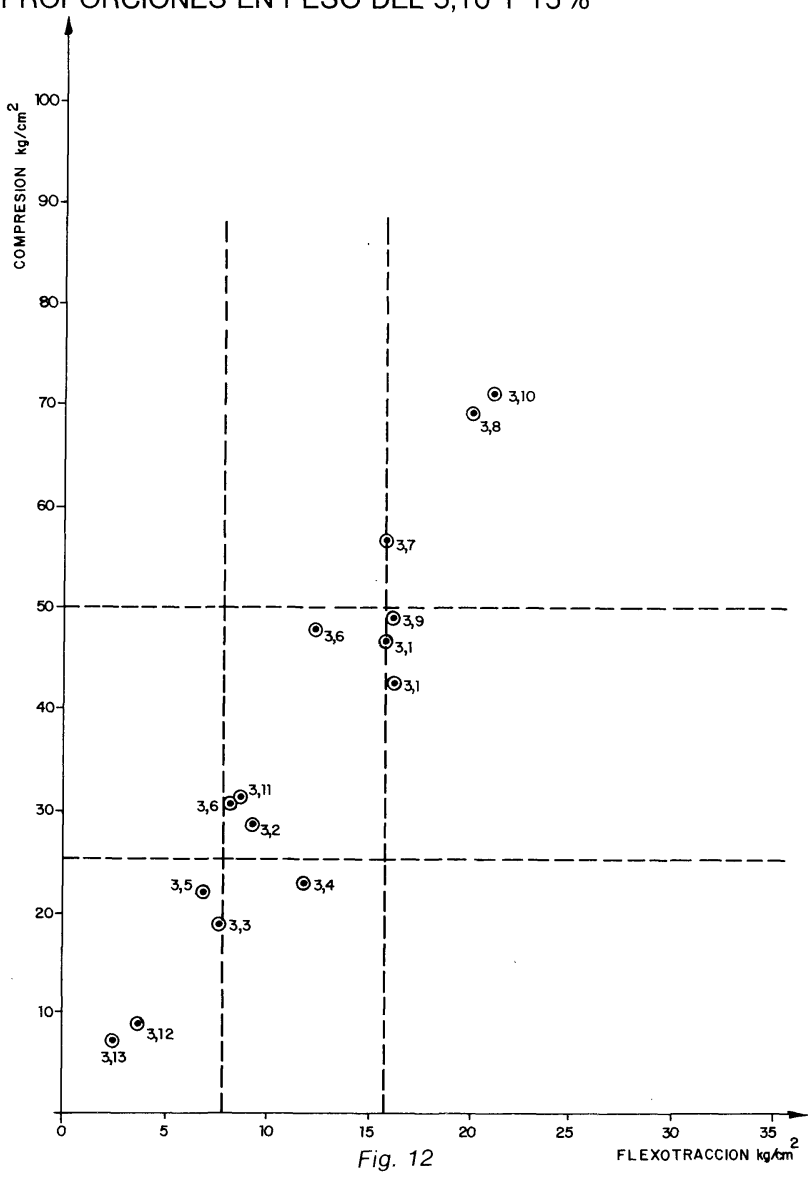

a) En el caso del suelo de Navapalos, todas las adiciones utilizadas perjudican desde el punto de vista resistente las características alcanzadas por el suelo primitivo.

Especialmente perjudicial se muestra la adición de cal, así como de la sustancia impermeabilizante a base de «Adogen», en las excesivas proporciones utilizadas.

b) En el caso del suelo de Alcalá-Chiloeches la adición de cenizas volantes en proporciones de $5 \% ; 10 \%$ y $15 \%$ así como las de $10 \%$ y $15 \%$ de cemento suponen mejoras, algunas de ellas sensibles, de las características resistentes alcanzadas por el suelo primitivo.

La adición de cal, bajas proporciones de cemento y la sustancia impermeabilizante utilizada, se muestran claramente perjudiciales.

\section{ENSAYO DE GOTEO}

Se han realizado baldosas de $15 \times 15 \times 2 \mathrm{~cm}$. de las 39 mezclas distintas especificadas en la Tabla n.o 4. El proceso seguido en la ejecución y secado de las baldosas ha sido el de la plasticidad normalizada. 


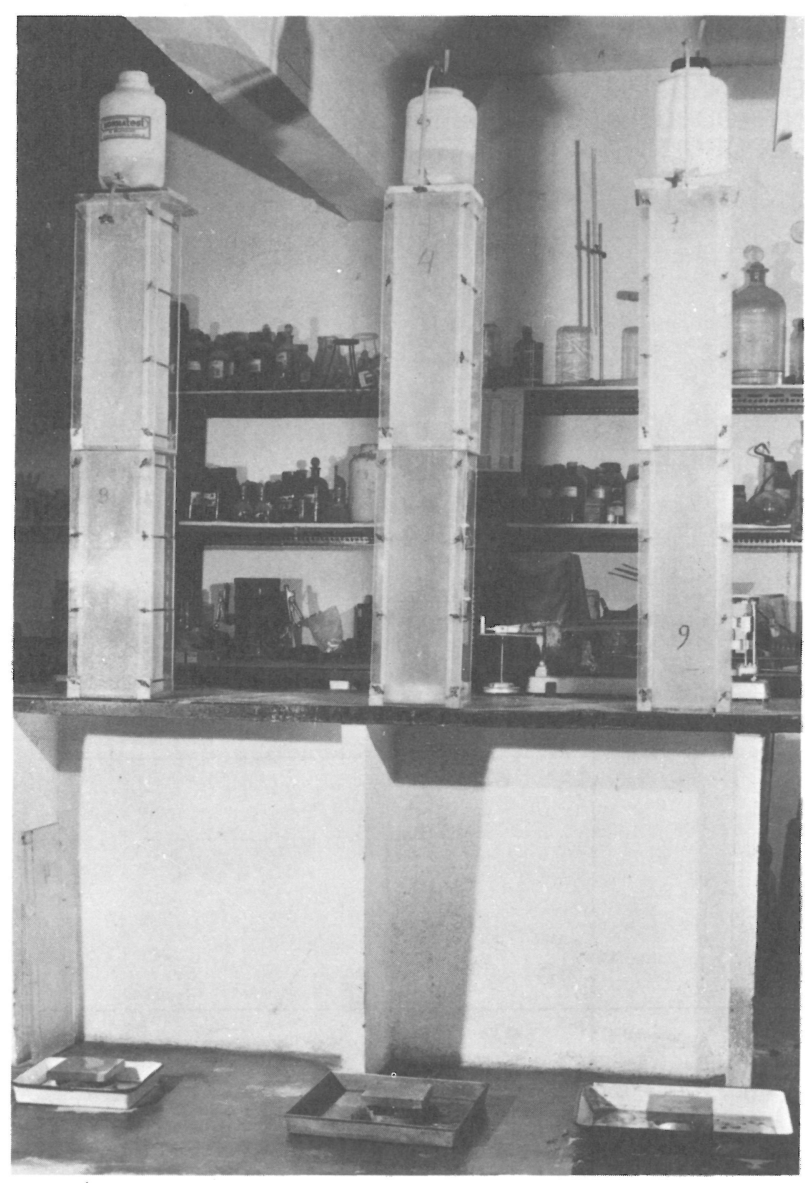

Fig. 13

Desde una altura de dos metros se dejó caer de forma constante agua en forma de gotas a un ritmo de $50 \div 60$ por minuto (lo que supone aproximadamente unos 0,416 litros/hora), tal y como

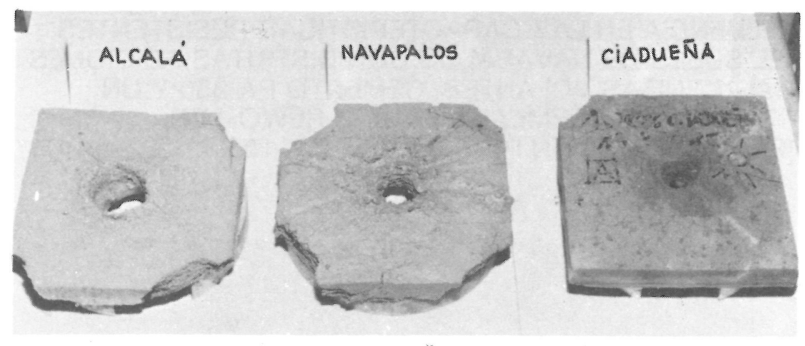

Fig. 14

se muestra en la fig. 13. Cada hora, durante las seis primeras, y posteriormente cada seis horas, se registraron sobre fichas preparadas al efecto los datos siguientes: tiempo hasta la aparición de huella; diámetro de huella; profundidad de huella; tiempo de perforación; tiempo de rotura y alteración o no de los bordes de la perforación (fig. 14).

Los ensayos, caso de no producirse previamente 'la perforación total, se mantuvieron durante 24 horas.

En la fig. 15 se han anotado en abscisas horas de duración del ensayo, y en ordenadas:

Parte inferior: Profundidad de la huella medida en milimetros desde 0 hasta $20 \mathrm{~mm}$, que corresponde a la perforación completa de la baldosa.

Parte superior: Diámetro medio de la huella desde 0 hasta $5 \mathrm{~cm}$.

Siguiendo con la idea de establecer niveles cualitativos, se han adoptado como comportamiento bajo, medio y alto frente a goteo los siguientes criterios. Se estima como bajo rendimiento de un

Tabla 8

ENSAYO de BALDOSAS al EXTERIOR.

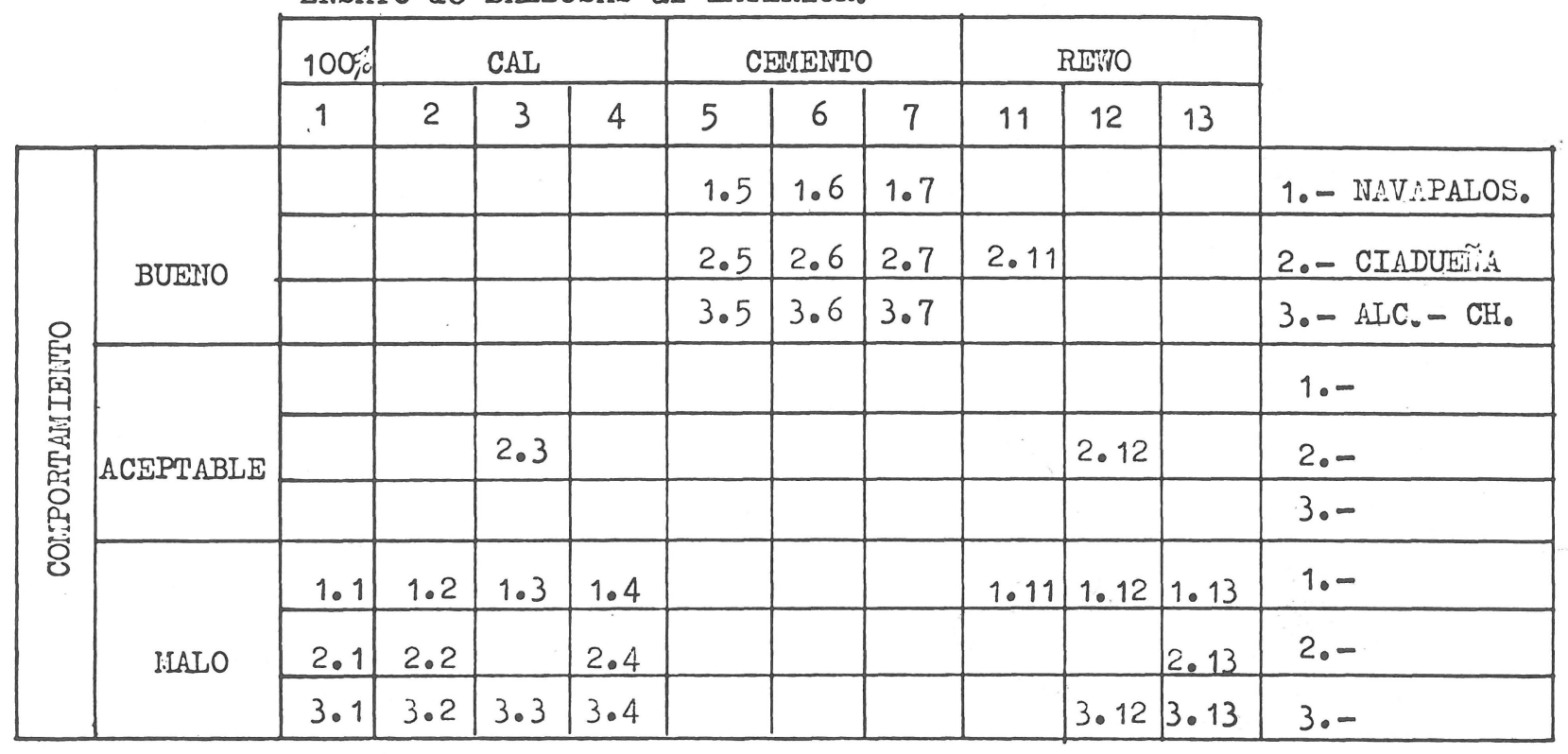




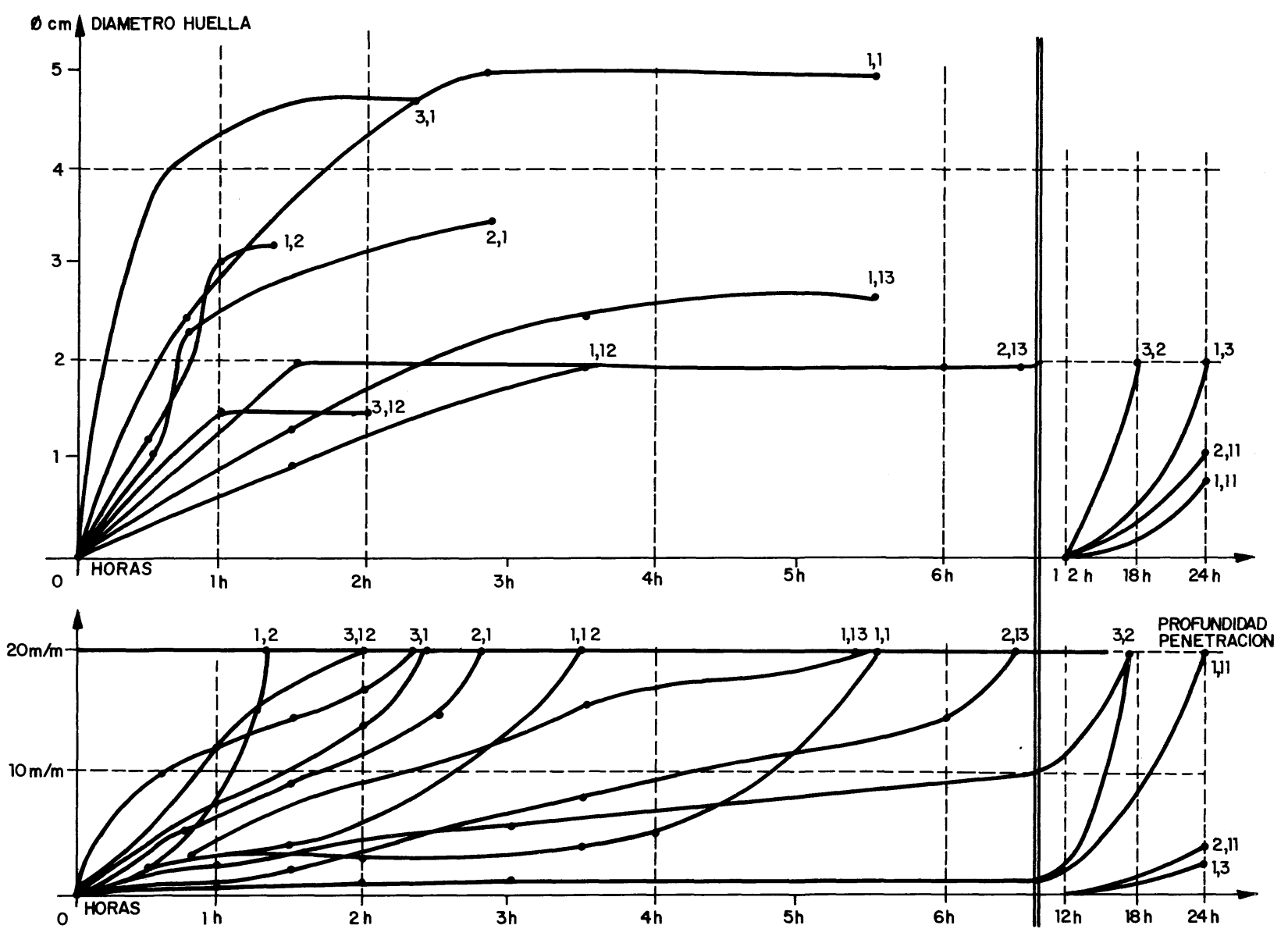

Fig. 15

suelo o mezcla frente a la acción del goteo, cuando se produce en las condiciones de ensayo descritas la perforación total de la baldosa antes de cuatro horas.

Rendimiento alto, cuando la perforación total no aparece en el transcurso de 24 horas de ensayo. En todos los supuestos descritos existen situaciones intermedias que se reflejan en la evolución de las curvas de la fig. 15 para los casos de bajo y medio rendimiento y en el tipo de huella final para las que superaron las 24 horas de exposición.

De acuerdo con estos criterios, en la Tabla 8 se recogen los resultados de las series de baldosas para suelos de Navapalos, Ciadueña y AlcaláChiloeches.

\section{ACCION CLIMATOLOGICA}

En el gráfico de la fig. 16 se representa el régimen climatológico de invierno en Madrid a que han sido expuestas 30 baldosas de $15 \times 15 \times 2$ $\mathrm{cm}$ elaboradas con las mismas dosificaciones que han sido objeto de estudio de comportamiento mecánico y a goteo, con la intención de establecer una relación entre éste y el correspondiente frente a los agentes atmosféricos en un clima que ofrece marcadas variaciones de temperatura, así como un notable régimen de precipitación media (fig. 17).

En el intervalo de tres meses de observación se establecen los niveles de: INALTERADAS, MICROFISURAS, FISURAS, GRIETAS y DERRUMBAMIENTO PARCIAL Y TOTAL, definiendo asi 6 parámetros que permitan relacionarlas con los niveles bajo, medio y alto anteriormente definidos, consiguiendo asi establecer niveles de calidad global.

A continuación se describen los diferentes estados/alteraciones que han ido adoptando cada una de las baldosas de forma detallada y los desórdenes más importantes observados en ellas, ya que debido a la variación entre máximos y mínimos, puntual de temperatura, precipitación de agua y nieve, e insolación, ha dado origen a cambios parciales representativos dentro del desarrollo global del ensayo. 
Con la intención de añadir datos a la información aportada, mediante la figura 18 puede seguirse la evolución cronológica del estado de las baldosas habiéndose establecido los siguientes niveles cualitativos de comportamiento:

\section{BAJO:}

- Dosificaciones de $100 \%$ de las 3 muestras de suelo (Ciadueña, Navapalos y Alcalá) (derrumbamiento).

- Mezclas de estos tres tipos de suelo con un contenido máximo de hasta $5 \%$ de cal.
- Mezclas de Alcalá y Navapalos con un contenido máximo del $10 \%$ de cal.

- Mezclas de Navapalos con contenidos del 5; 10 y $15 \%$ de Adogen.

- Ciadueña más 10\% de Adogen.

En todas ellas se ha producido en el tiempo considerado y con las condiciones climatológicas expresadas un acusado desgaste, estado de fisuración superficial generalizado, llegando a producirse en algunas de ellas desprendimientos parciales e incluso derrumbamiento total.

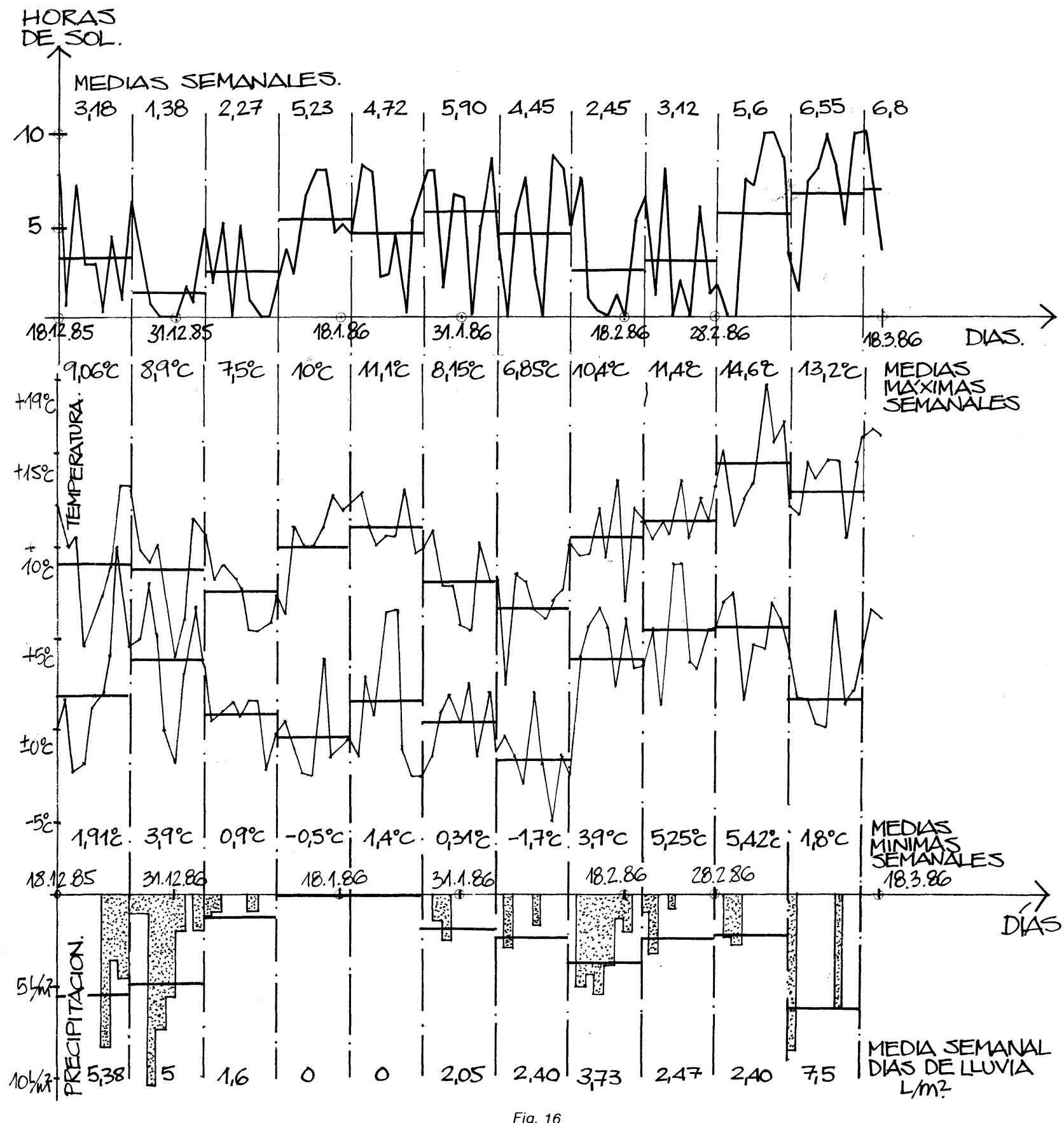




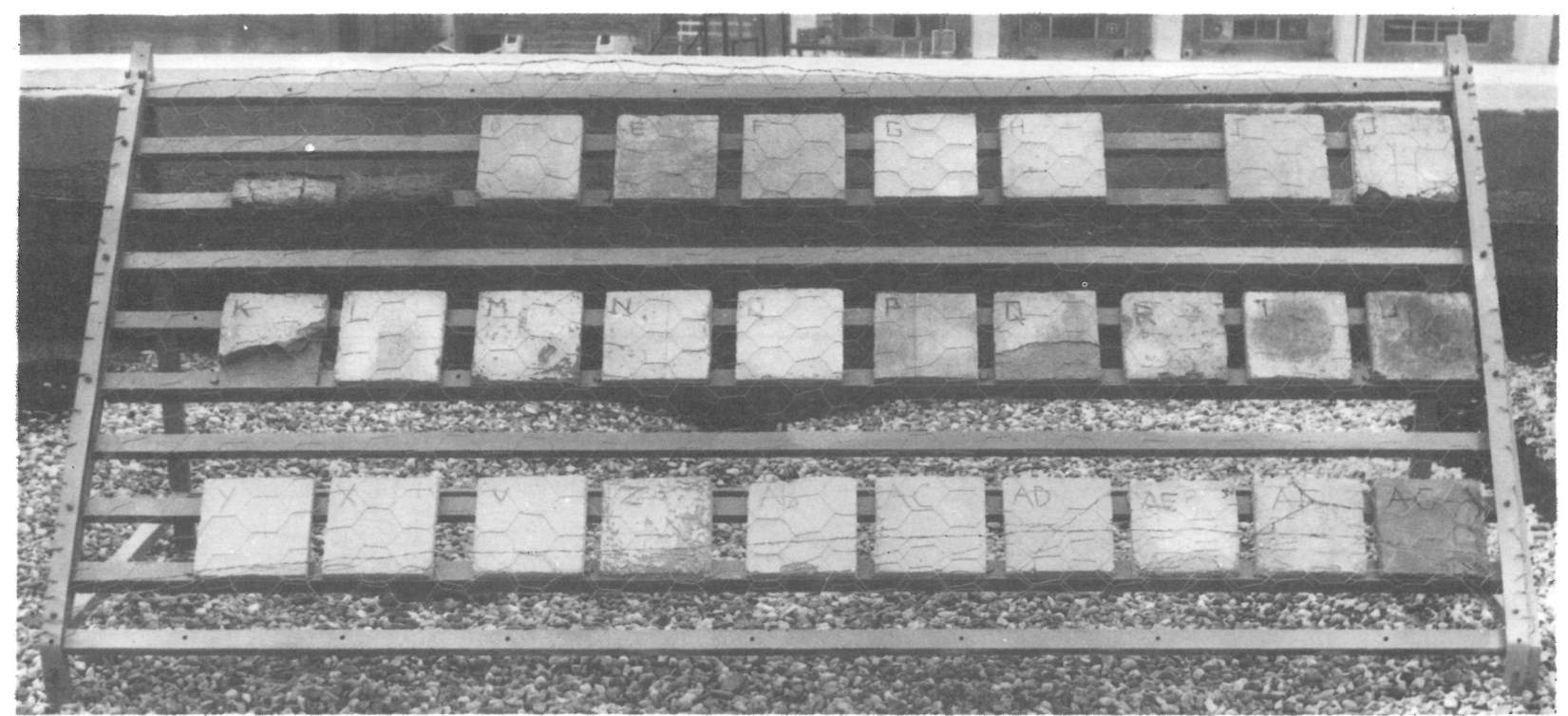

Fig. 17.-Situación de las baldosas durante el ensayo.

Tabla 9

\begin{tabular}{|c|c|c|c|c|c|c|c|c|c|c|c|c|c|c|c|}
\hline \multicolumn{3}{|c|}{ PROCEDENCIA } & \multicolumn{13}{|c|}{ COMPOSICION } \\
\hline 1 & 2 & 3 & 1 & 2 & 3 & 4 & 5 & 6 & 7 & 8 & 9 & 10 & 11 & 12 & 13 \\
\hline \multirow{8}{*}{ 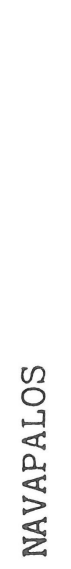 } & \multirow{8}{*}{  } & \multirow{8}{*}{ 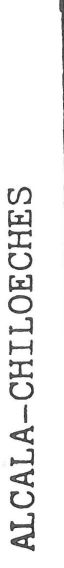 } & \multirow{8}{*}{ 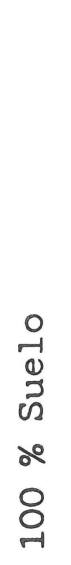 } & స్ర & 0 & ల్ & $\begin{array}{l}\stackrel{\bigcirc}{+} \\
\stackrel{0}{E} \\
\stackrel{0}{E}\end{array}$ & 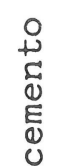 & 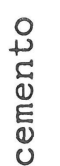 & $\begin{array}{l}\mathbb{N} \\
\stackrel{N}{\widetilde{E}} \\
\mathbb{0}\end{array}$ & $\begin{array}{l}\text { N } \\
\text { न } \\
\tilde{d} \\
0 \\
0\end{array}$ & $\begin{array}{l}\stackrel{\mathbb{N}}{N} \\
\stackrel{\sim}{\mathbb{D}} \\
0 \\
0\end{array}$ & 总 &  & 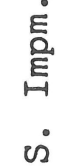 \\
\hline & & & & 0 & ১ீ & ஃீ & \begin{tabular}{l}
0 \\
\hdashline
\end{tabular} & xீ & ஃ゚ & 0 & ฉீ & ஓீ & అ & ๖ீ & ஃீ \\
\hline & & & & ๘ீ & 아 & $\ln _{-1}^{n}$ & 2 & 욱 & $\stackrel{n}{\rightarrow-1}$ & ๘ & 우 & $\stackrel{n}{\sim}$ & م & 악 & $\stackrel{n}{\sim}$ \\
\hline & & & & + & + & + & + & + & + & + & + & + & + & + & + \\
\hline & & & & $\underset{\sim}{\circ}$ & $\stackrel{ }{\circ}$ & 엇 & 억 & $\stackrel{0}{-1}$ & $\stackrel{0}{\rightarrow-1}$ & $\stackrel{0}{-1}$ & $\underset{-1}{0}$ & $\underset{-1}{0}$ & $\stackrel{0}{-1}$ & 욱 & 엄 \\
\hline & & & & ગ & ڤ్ల & गे & 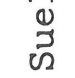 & $\stackrel{0}{J}$ & $\begin{array}{l}\stackrel{0}{J} \\
\text { బ }\end{array}$ & $\stackrel{0}{\mathcal{~}}$ & $\stackrel{0}{\mathcal{J}}$ & $\stackrel{0}{コ}$ & $\stackrel{\circlearrowright}{コ}$ & $\stackrel{0}{\mathcal{D}}$ & 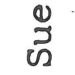 \\
\hline & & & & ஃீ & de & de & × & de & ๖ீ & শ゚ & de & x & 20 & ๙ீ & de \\
\hline & & & & $\stackrel{\leftrightarrow}{\circ}$ & ৪ & $\infty$ & ต̊ & ৪ & $\stackrel{\llcorner}{\infty}$ & ค็ & ৪ & $\mathscr{\infty}$ & $\stackrel{\llcorner}{\circ}$ & ৪ & $\stackrel{\infty}{\infty}$ \\
\hline \multicolumn{3}{|c|}{ SUELO } & & $\mathrm{CON}$ & $\mathrm{CA}$ & & $\mathrm{CON}$ & CEME & JTO & $\mathrm{CON}$ & $\mathrm{CE}$ & IZA & CON & YPEF & MEA. \\
\hline
\end{tabular}

MEDIO:

- Dosificación con un contenido máximo de hasta $15 \%$ de cal en los tres tipos de suelo estudiados.

- Ciadueña con un contenido del $10 \%$ de cal ó de $10 \%$ y $15 \%$ de Adogen.

- Navapalos más $5 \%$ Adogen.
ALTO:

Dosificaciones con contenidos de 5; 10 y $15 \%$ de cemento PA-350 en los tres tipos de suelo en los que se puede considerar que han tenido una alteración inapreciable.

En la Tabla n.o 9 y siguiendo la sistemática general, se recogen los distintos niveles de comportamiento cualitativo de las 39 baldosas sometidas a la acción climatológica. 


\section{EVOLUCION DE LAS ALTERACIONES DE LAS BALDOSAS A LO LARGO DEL TIEMPO}

Fecha de comienzo del ensayo 18-12-85

La nomenclatura empleada se corresponde con la establecida en la Tabla-4. Su posición de las baldosas en la figura es la que tienen durante el ensayo. Ver fig. 17

\begin{tabular}{|c|c|c|c|c|c|c|c|c|c|c|}
\hline \multicolumn{3}{|c|}{ CLAVE DE SIMBOLOS: } & \multicolumn{4}{|c|}{$\begin{array}{l}\text { - sin alteración } \\
+ \text { microfisuras } \\
\triangle \text { fisuras }\end{array}$} & \multicolumn{4}{|c|}{$\begin{array}{l}\square \text { grietas } \\
0 \text { derrumbamiento parcial } \\
* \text { derrumbamiento total }\end{array}$} \\
\hline \multirow{2}{*}{$\begin{array}{c}\text { Toma de } \\
\text { datos }\end{array}$} & \multicolumn{2}{|c|}{ Baldosa: } & \multirow[b]{2}{*}{2.5} & \multirow[b]{2}{*}{3.5} & \multirow[b]{2}{*}{2.6} & \multirow[b]{2}{*}{3.6} & \multirow[b]{2}{*}{3.7} & \multirow[b]{2}{*}{2.7} & \multirow[b]{2}{*}{3.2} & \\
\hline & 1.1 & 3.1 & & & & & & & & \\
\hline $23-12-85$ & $\triangle$ & $\triangle$ & - & - & - & - & - & - & - & \\
\hline $30-12-85$ & $*$ & $*$ & - & - & - & - & - & - & + & \\
\hline 7- 1-86 & & & - & - & - & - & - & - & $\triangle$ & \\
\hline $13-1-86$ & & & - & - & - & - & - & + & $\square$ & \\
\hline $20-1-86$ & & & - & - & - & - & + & + & $\square$ & \\
\hline $27-1-86$ & & & - & - & - & - & + & + & $\square$ & \\
\hline 3- $2-86$ & & & - & - & - & - & + & + & $\square$ & \\
\hline $10-2-86$ & & & - & - & - & - & + & + & $\square$ & \\
\hline $17-2-86$ & & & - & - & - & - & + & + & $\overline{0}$ & \\
\hline 24- 2-86 & & & - & - & - & - & + & + & 0 & \\
\hline 3- 3-86 & & & - & - & - & - & + & + & 0 & \\
\hline $10-3-86$ & & & - & - & - & - & + & + & 0 & \\
\hline $17-3-86$ & & & - & - & - & + & + & + & 0 & \\
\hline \multirow{2}{*}{$\begin{array}{c}\text { Toma } \\
\text { de datos }\end{array}$} & & & & & & & & & & \\
\hline & 3.3 & 3.4 & 2.2 & 2.3 & 2.4 & 2.11 & 3.12 & 3.13 & 2.12 & 2.13 \\
\hline $23-12-85$ & + & -- & + & + & - & - & - & - & - & - \\
\hline $30-12-85$ & + & + & + & + & - & - & + & + & + & + \\
\hline 7- 1-86 & $\triangle$ & $\triangle$ & $\triangle$ & + & - & - & $\triangle$ & $\triangle$ & + & $\triangle$ \\
\hline 13- $1-86$ & $\square$ & $\triangle$ & $\square$ & $\triangle$ & $\triangle$ & + & $\triangle$ & $\square$ & $\triangle$ & $\square$ \\
\hline $20-1-86$ & $\square$ & $\triangle$ & $\square$ & $\triangle$ & $\triangle$ & + & $\triangle$ & 0 & $\triangle$ & $\square$ \\
\hline 27- $1-86$ & $\square$ & $\triangle$ & $\square$ & $\triangle$ & $\triangle$ & + & $\triangle$ & 0 & $\triangle$ & $\square$ \\
\hline 3- $2-86$ & $\square$ & $\triangle$ & $\square$ & $\triangle$ & $\triangle$ & + & $\triangle$ & 0 & $\triangle$ & $\square$ \\
\hline $10-2-86$ & $\square$ & $\triangle$ & $\square$ & $\triangle$ & $\triangle$ & + & $\bar{\Delta}$ & 0 & $\triangle$ & $\square$ \\
\hline $17-2-86$ & 0 & $\triangle$ & $\square$ & $\triangle$ & $\triangle$ & + & $\square$ & 0 & $\triangle$ & $\square$ \\
\hline 24- $2-86$ & 0 & $\triangle$ & $\square$ & $\triangle$ & $\triangle$ & + & 0 & 0 & $\triangle$ & $\square$ \\
\hline 3- 3-86 & 0 & $\triangle$ & $\square$ & $\triangle$ & $\triangle$ & + & 0 & 0 & $\triangle$ & $\square$ \\
\hline $10-3-86$ & 0 & $\square$ & $\square$ & $\triangle$ & $\square$ & + & 0 & $*$ & $\triangle$ & $\square$ \\
\hline $17-3-86$ & 0 & $\square$ & $\square$ & $\triangle$ & $\square$ & + & 0 & $*$ & $\triangle$ & $\square$ \\
\hline \multirow{2}{*}{$\begin{array}{c}\text { Toma de } \\
\text { datos }\end{array}$} & & & & & & & & & & \\
\hline & 1.5 & 1.6 & 1.7 & 1.2 & 1.3 & 1.4 & 1.11 & 1.12 & 1.13 & 1.14 \\
\hline $23-12-85$ & - & - & - & - & - & - & - & - & - & - \\
\hline $30-12-85$ & - & - & - & + & + & - & - & + & + & + \\
\hline 7- 1-86 & - & - & - & $\triangle$ & $\triangle$ & + & + & $\triangle$ & $\triangle$ & $\triangle$ \\
\hline $13-1-86$ & - & - & - & $\square$ & $\square$ & $\triangle$ & $\triangle$ & $\square$ & $\square$ & $\square$ \\
\hline $20-1-86$ & - & - & - & $\square$ & $\square$ & $\triangle$ & $\triangle$ & $\square$ & $\square$ & $\square$ \\
\hline 27- $1-86$ & - & - & - & $\square$ & $\square$ & $\triangle$ & $\triangle$ & $\square$ & $\square$ & $\square$ \\
\hline 3- $2-86$ & - & - & - & $\square$ & $\square$ & $\triangle$ & $\triangle$ & $\square$ & $\square$ & $\square$ \\
\hline $10-2-86$ & - & - & - & $\square$ & $\square$ & $\triangle$ & $\triangle$ & $\square$ & $\square$ & $\square$ \\
\hline $17-2-86$ & - & - & - & $\square$ & $\square$ & $\square$ & $\triangle$ & 0 & 0 & 0 \\
\hline 24- $2-86$ & + & - & - & $\square$ & 0 & $\square$ & $\square$ & 0 & 0 & 0 \\
\hline 3- 3-86 & $\triangle$ & - & - & $\square$ & 0 & $\square$ & $\square$ & 0 & 0 & 0 \\
\hline $10-3-86$ & $\triangle$ & - & - & $\square$ & 0 & $\square$ & $\square$ & 0 & 0 & 0 \\
\hline $17-3-86$ & $\Delta$ & - & - & $\square$ & 0 & $\square$ & $\square$ & 0 & 0 & 0 \\
\hline
\end{tabular}




\section{BIBLIOGRAFIA}

- La durabilite de protections sur terre stabilisee: L'experience de “Murets Dreyfus» de Dakar. J. P. Laurent, Rexcoop. CSTB, féb. 1985.

- Caracterisation des Briques de terre crue compresse par L'essai Proctor Statique. Rapport final, Myriam Olivier, diciembre 1985, Rexcoop.

- Resistencia sísmica de la mamposteria de adobe, Julio Vagas Neumann, Juan Barriola Bernales, Marcial Blondet. Pontificia Universidad Católica del Perú, abril 1984.

- Actualité de la construction de terre en France, Actes du seminaire, Plan construction, 14-15 de octubre 1982.

- Essais simplifies pour l'identification et la qualification des terres a construire. Rapport final, Ph. Michel, D. Simon, Plan Construction, mayo 1985.

- Constructions en terre stabilisee en Cote D'Ivore bilanet perspectives. Abraham Quattara LBTP, Simon- net Jacques LBTP. Proceedings of Symposium en Nairobi, Kenya, 7-14 nov. 1983.

- Etude exploratoire de quelques techniques de stabilisation chimique de la terre. Philippe Eurin, Marcel Rubaud. Centre Scientifique et Technique du Bâtiment, Grenoble. Proceedings of Symposium en Nairobi, Kenya, 7-14 nov. 1983.

- Programme experimental sur badigeons de protection des murs en béton de terre. D. Mariotti, Délégué Technique Extérieures au C.E.B.T.P. Proceedings of Symposium en Nairobi, Kenya, 7-14 nov. 1983.

- Construire en Terre, Craterre. Paris, 1983.

- Lime stabilisation of clay soi 1. Gallaway, B. M.; Buchanan, S. J. Agricultural and Mechanical College of Texas, 1951.

- Gernot Minke, LOW-COST - Bauen, Forschungsbericht, DFG Mi 147/3 y 3, Forschungslabor für Experimentelles Bauen, Gesamthochschule Kassel, 1980.

\section{AGRADECIMIENTO}

Queremos agradecer la colaboración y apoyo prestado a este trabajo por científicos como D. a Isabel Sánchez Rojas y D. José Luis Sagrera del I.E.T.c.c., D. Antonio García Verduch, D. Francisco José Vallé y D. a Isabel Nieto del Instituto de Cerámica y Vidrio y D. a Covadonga Rodríguez del Instituto de Edafología, todos ellos del Consejo Superior de Investigaciones Cientificas.

Como responsable del Proyecto de Investigación «Materiales, Tecnologías y Prototipos de Viviendas de Muy Bajo Coste", deseo dejar constancia de mi sincero agradecimiento, por el trabajo intenso, entusiasta y colectivo que han desarrollado todos los componentes del equipo (M. Alvarez, P. Díaz Romeral, E. Estrada, M.a J. Guinea, C. Luengo, O. Martín, E. Rohmer, P. Valiente y J. Veras) a lo largo de la preparación de las JORNADAS "La Tierra, material de construcción", ellos se han ocupado desde la realización de adobes, a la redacción de ponencias, sin descuidar el montaje de las exposiciones.

El que este número de INFORMES salga a la luz en un plazo ajustado, ha sido posible gracias a la dedicación ejemplar de M. Martínez Mallén y R. Irigoyen. A ambos mi reconocimiento. 